\title{
Exact analytic results for the Gutzwiller wave function with finite magnetization
}

\author{
Marcus Kollar* and Dieter Vollhardt ${ }^{\dagger}$ \\ Theoretische Physik III, Elektronische Korrelationen und Magnetismus, Institut für Physik, Universität Augsburg, \\ D-86135 Augsburg, Germany
}

(Received 7 November 2001; published 12 April 2002)

\begin{abstract}
We present analytic results for ground-state properties of Hubbard-type models in terms of the Gutzwiller variational wave function with nonzero values of the magnetization $m$. In dimension $D=1$ approximation-free evaluations are made possible by appropriate canonical transformations and an analysis of umklapp processes. We calculate the double occupation and the momentum distribution, as well as its discontinuity at the Fermi surface, for arbitrary values of the interaction parameter $g$, density $n$, and magnetization $m$. These quantities determine the expectation value of the one-dimensional Hubbard Hamiltonian for any symmetric, monotonically increasing dispersion $\epsilon_{k}$. In particular for nearest-neighbor hopping and densities away from half filling the Gutzwiller wave function is found to predict ferromagnetic behavior for sufficiently large interaction $U$.
\end{abstract}

DOI: 10.1103/PhysRevB.65.155121

PACS number(s): 71.27.+a

\section{INTRODUCTION}

Quantum-mechanical many-body problems can almost never be solved exactly. In this situation variational wave functions have proved to be particularly useful. Although they describe correlations among the particles only in an approximate way, they have the advantage of being explicit and physically intuitive. In particular, they allow for investigations even when standard perturbation theory is not applicable, or is untractable.

Variational wave functions can, for example, be obtained by applying a suitably chosen correlation operator (e.g., the interaction part of the Hamiltonian under investigation) to a simple one-particle wave function. For the one-band Hubbard model ${ }^{1-3}$

$$
\hat{H}=\sum_{k \sigma} \epsilon_{k} \hat{a}_{k \sigma}^{+} \hat{a}_{k \sigma}+U \sum_{i} \hat{n}_{i \uparrow} \hat{n}_{i \downarrow}
$$

which is often used as an effective model to understand electronic correlation phenomena like itinerant ferromagnetism in transition metals, high-temperature superconductivity, and the Mott-Hubbard metal-insulator transition, the simplest projected wave function is the Gutzwiller wave function (GWF), ${ }^{3}$

$$
\left|\Psi_{\mathrm{G}}\right\rangle=g^{\Sigma_{i} \hat{D}_{i}}\left|\Phi_{0}\right\rangle=\prod_{i}\left[1-(1-g) \hat{D}_{i}\right]\left|\Phi_{0}\right\rangle
$$

where $g$ is a variational parameter (usually $0 \leqslant g \leqslant 1$ ), $\hat{D}_{i}$ $=\hat{n}_{i \uparrow} \hat{n}_{i \downarrow}$ is the operator for double occupation at lattice site $i$, and the starting wave function $\left|\Phi_{0}\right\rangle$ is a product state of spin-up and spin-down Fermi seas,

$$
\left|\Phi_{0}\right\rangle=\prod_{\substack{k \sigma \\ \epsilon_{k} \leqslant \epsilon_{\mathrm{F} \sigma}}} \hat{a}_{\boldsymbol{k} \sigma}^{+}|\mathrm{vac}\rangle .
$$

Using the GWF one may, in principle, calculate expectation values of any operator $\hat{A}$ as $\langle\hat{A}\rangle_{\mathrm{G}}=\left\langle\Psi_{\mathrm{G}}|\hat{A}| \Psi_{\mathrm{G}}\right\rangle /\left\langle\Psi_{\mathrm{G}} \mid \Psi_{\mathrm{G}}\right\rangle$.
By the variational principle the energy expectation value $\langle\hat{H}\rangle_{\mathrm{G}}$ is an upper bound for the true ground-state energy of $\hat{H}$.

The properties and quality of the GWF have been the subject of detailed investigations (for an early review, see Ref. 4). A diagrammatic theory for the calculation of expectation values in terms of the GWF, valid in arbitrary dimensions $D$, was formulated by Metzner and Vollhardt. ${ }^{5}$ (Reference 5 is hereafter referred to as MV.) In particular, for systems without net magnetic polarization $m=n_{\uparrow}-n_{\downarrow}=0$, i.e., particle densities $n_{\sigma}=n / 2$ with $k_{\mathrm{F} \uparrow}=k_{\mathrm{F} \downarrow}$ in Eq. (3), they calculated the momentum distribution $n_{k \sigma}=\left\langle\hat{a}_{k \sigma}^{+} \hat{a}_{k \sigma}\right\rangle_{\mathrm{G}}$ and the double occupation $d=\left\langle\sum_{i} \hat{D}_{i}\right\rangle_{\mathrm{G}} / L$ analytically in $D=1$ for all values of $g$ and $n$, where $L$ is the number of lattice sites. The analytic calculation of correlation functions, in particular of the spin-spin correlation function, by Gebhard and Vollhardt ${ }^{6}$ showed that in the nonmagnetic case, for $U$ $\rightarrow \infty$, the results obtained with the GWF are in very good agreement with exact analytic and numerical results for the antiferromagnetic Heisenberg chain. In fact, Haldane ${ }^{7}$ and Shastry ${ }^{8}$ discovered that the GWF at $g=0$ is the exact ground-state wave function of the antiferromagnetic Heisenberg chain with $1 / r^{2}$ exchange. Results were also obtained in dimensions $D=1,2,3$ using numerical techniques ${ }^{9,10}$ and finite orders of perturbation theory. ${ }^{11,12}$ Within the diagrammatic approach of $\mathrm{MV}$ it also became possible to derive the well-known Gutzwiller approximation in the limit of infinite spatial dimensions $(D=\infty) .{ }^{13}$ Comprehensive investigations in this limit were made possible by the approach of Gebhard ${ }^{14}$ which allows for explicit evaluations of expectation values for arbitrary starting wave functions $\left|\Phi_{0}\right\rangle$ (including ones with broken symmetry) and facilitates the expansion in $1 / D$ around $D=\infty$. This approach was also extended to multiband Hubbard models; ${ }^{15}$ recently that method was combined with density-functional theory, and applied to ferromagnetic transition metals. ${ }^{16}$ The Gutzwiller approximation also describes a correlation-induced transition from metal to insulator, the Brinkman-Rice transition. ${ }^{17} \mathrm{We}$ recently investigated the effect of correlated hopping, which 
for the GWF can be calculated in terms of $n_{k \sigma}$ and $d$ in any dimension, on this transition. ${ }^{18}$

Up to now the analytic calculation of expectation values in $D=1$ was limited to the unmagnetized paramagnetic phase. In view of the renewed interest in the microscopic foundations of metallic ferromagnetism (see Ref. 19 for a review), it is desirable to perform such evaluations also for the GWF with nonzero magnetization $(m \neq 0)$. In this paper we show that, in spite of formidable technical complications, it is indeed possible to evaluate such expectation values even for finite magnetization. From suitable canonical transformations we obtain diagrammatic relations and reduce the expectation values for $m \neq 0$ to those for $m=0$. Thereby it becomes possible to calculate the double occupation $d$ and the momentum distribution $n_{k \sigma}$ for arbitrary values of the correlation parameter $g$, density $n$, and magnetization $m$. Furthermore we derive an expression for $n_{k \sigma}$ in closed form, which was not available up to now even for zero magnetization. These quantities determine the energy expectation value and thus the optimal variational parameter and spontaneous magnetization.

The paper is structured as follows: In Sec. II the diagrammatic formulation is used to derive diagrammatic relations from canonical transformations, valid in arbitrary dimensions. The evaluation of expectation values is derived in Sec. III. The resulting magnetic phase diagram for the Hubbard model in $D=1$ is presented in Sec. IV. The conclusion in Sec. $\mathrm{V}$ closes the presentation.

\section{DIAGRAMMATIC FORMULATION IN ARBITRARY DIMENSION $D$}

\section{A. General formalism}

The double occupation $d(g, n, m)$ and the momentum distribution $n_{k \sigma}(g, n, m)$ of the GWF are required for the calculation of the variational energy, $E_{\mathrm{G}}=\langle\hat{H}\rangle_{\mathrm{G}} / L$. Another quantity of interest is the discontinuity $q_{\sigma}$ of $n_{k \sigma}$ at the Fermi surface, $q_{\sigma}(g, n, m)=n_{k_{\mathrm{F} \sigma}^{-} \sigma}(g, n, m)-n_{k_{\mathrm{F} \sigma}^{+} \sigma}(g, n, m) .{ }^{20}$ The rules for the diagrammatic expansion of these expectation values in powers of $\left(g^{2}-1\right)$ were developed by MV, with the result

$$
\begin{gathered}
d(g, n, m)=g^{2} \sum_{p=1}^{\infty}\left(g^{2}-1\right)^{p-1} c_{p}(n, m), \\
n_{k \sigma}(g, n, m)=\left[1-(1-g)^{2} n_{-\sigma}\right] n_{k \sigma}^{0} \\
+\frac{1-\left(1-g^{2}\right) n_{k \sigma}^{0}}{(1+g)^{2}} \sum_{p=2}^{\infty}\left(g^{2}-1\right)^{p} f_{p \sigma}(\boldsymbol{k}, n, m),
\end{gathered}
$$

where $n_{k \sigma}^{0}=n_{k \sigma}(g=1, n, m)$. The functions $c_{p}(n, m)$ and $f_{p \sigma}(\boldsymbol{k}, n, m)$ can be represented by Feynman diagrams corresponding to those of the energy and the Green's function, respectively, of a $\phi^{4}$ theory. For later convenience we define

$$
f_{1 \sigma}=-n_{-\sigma} n_{k \sigma}^{0}, \quad f_{0 \sigma}=n_{k \sigma}^{0}, \quad c_{0}=-\frac{n-|m|}{2} .
$$

The diagrams for $c_{p}(n, m)$ can be obtained from those for $f_{p \sigma}(\boldsymbol{k}, n, m)$ by connecting the two external vertices (see $\mathrm{MV})$ :

$$
c_{p}(n, m)=-\frac{1}{L} \sum_{k} n_{k \sigma}^{0} f_{p \sigma}(\boldsymbol{k}, n, m), \quad p \geqslant 1 .
$$

This equation yields sum rules ${ }^{21}$ for the density of particles inside, $n_{\sigma}^{<}$, or outside of the Fermi surface, $n_{\sigma}^{>}$, namely

$$
\begin{gathered}
n_{\sigma}^{<}=n_{\sigma}-n_{\sigma}^{>}=\frac{1}{L} \sum_{k} n_{k \sigma}^{0} n_{k \sigma}, \\
n_{\uparrow}^{>}=n_{\downarrow}^{>}=\frac{1-g}{1+g}\left(\frac{n^{2}-m^{2}}{4}-d(g, n, m)\right) .
\end{gathered}
$$

It is sometimes useful to remove the diagrams $c_{p}$ from $f_{p \sigma}$ and thus define

$$
h_{p \sigma}(\boldsymbol{k}, n, m)=f_{p \sigma}(\boldsymbol{k}, n, m)-c_{p-1}(n, m), \quad p \geqslant 1 .
$$

Note that our definitions in Eqs. (5) and (9) differ slightly from MV.

\section{B. Canonical transformations}

For the relations to be discussed next the hopping amplitude $t_{i j}$ is assumed to be nonzero only for hopping between sites $i$ and $j$ on different sublattices $A$ and $B$. In the next chapter we will see, however, that in dimension $D=1$ this requirement can be dropped. The simplest canonical transformation is the interchange of spin indices $\uparrow$ and $\downarrow$ which implies

$$
\begin{gathered}
d(g, n, m)=d(g, n,-m), \\
n_{k \sigma}(g, n, m)=n_{\boldsymbol{k}-\sigma}(g, n,-m) .
\end{gathered}
$$

Furthermore, a particle-hole transformation for both spins yields (MV)

$$
\begin{gathered}
d(g, n, m)=d(g, 2-n,-m)+n-1, \\
n_{\boldsymbol{k} \sigma}(g, n, m)=1-n_{\boldsymbol{Q}-\boldsymbol{k} \sigma}(g, 2-n,-m) .
\end{gathered}
$$

Here $\boldsymbol{Q}$ is a vector in the first Brillouin zone with $e^{\mathrm{i} \boldsymbol{Q} \cdot \boldsymbol{R}}$ $= \pm 1$ for a lattice vector $\boldsymbol{R} \in A, B$, respectively; $\boldsymbol{Q}$ $=(\pi / a, \pi / a, \cdots \pi / a)$ for a hypercubic lattice with spacing a. From Eqs. (10b) and (11b) it follows that the discontinuity at the Fermi surface obeys ${ }^{20}$

$$
q_{\sigma}(g, n, m)=q_{-\sigma}(g, n,-m)=q_{-\sigma}(g, 2-n, m) .
$$

Note that in particular $q_{\uparrow}=q_{\downarrow}$ for $n=1$.

For densities $0 \leqslant n \leqslant 2$ the magnetization is in the range $|m| \leqslant \min (n, 2-n)$. In view of Eqs. (10)-(12) we limit ourselves from now on to $0 \leqslant m<n \leqslant 1$. Therefore $\sigma=\uparrow$ will be referred to as "majority spin" and $\sigma=\downarrow$ as "minority spin." 
Note that the case $n=m$ (the fully polarized state without doubly occupied sites) can be obtained from the uncorrelated case $g=1$.

Performing a particle-hole transformation for $\uparrow$ operators only, ${ }^{6}$

$$
\hat{c}_{i \uparrow}^{\prime}=(-1)^{i} \hat{c}_{i \uparrow}^{+}, \quad \hat{c}_{i \downarrow}^{\prime}=\hat{c}_{i \downarrow},
$$

one may derive the following identities for $d(g, n, m)$ and $n_{k \sigma}(g, n, m)$ :

$$
\begin{gathered}
d(g, n, m)=\frac{n-m}{2}-d\left(g^{-1}, 1-m, 1-n\right) . \\
n_{\boldsymbol{k} \uparrow}(g, n, m)=1-n_{Q-k \uparrow}\left(g^{-1}, 1-m, 1-n\right), \\
n_{\boldsymbol{k} \downarrow}(g, n, m)=n_{\boldsymbol{k} \downarrow}\left(g^{-1}, 1-m, 1-n\right) .
\end{gathered}
$$

For the uncorrelated case $(g=1)$ we have in particular

$$
\begin{gathered}
n_{\boldsymbol{k} \uparrow}^{0}(n, m)=1-n_{\boldsymbol{Q}-\boldsymbol{k} \uparrow}^{0}(1-m, 1-n), \\
n_{\boldsymbol{k} \downarrow}^{0}(n, m)=n_{\boldsymbol{k} \downarrow}^{0}(1-m, 1-n) .
\end{gathered}
$$

The relations in Eq. (15) express a property of the starting wave function [Eq. (3)] and can also be derived directly from the fact that hopping occurs only between $A$ and $B$ sublattices.

\section{Diagrammatic relations}

We now derive diagrammatic relations for $c_{p}$ and $f_{p \sigma}$ from the identities in Eqs. (14) and (15). The following equations are valid for all $p \geqslant 0$ and $0 \leqslant m \leqslant n \leqslant 1$ unless noted otherwise.

\section{Double occupation}

From Eq. (4a) we obtain

$$
\begin{aligned}
& d\left(g^{-1}, 1-m, 1-n\right) \\
& \quad=\sum_{p=0}^{\infty}\left(g^{2}-1\right)^{p}(-1)^{p}\left(\frac{1}{g^{2}}\right)^{p+1} c_{p+1}(1-m, 1-n) .
\end{aligned}
$$

Our goal is to equate coefficients of powers of $\left(g^{2}-1\right)$. To this end we make use of the expansion

$$
\left(\frac{1}{g^{2}}\right)^{p+1}=\sum_{r=0}^{\infty}\left(\begin{array}{c}
-p-1 \\
r
\end{array}\right)\left(g^{2}-1\right)^{r} .
$$

We then obtain from Eq. (14a)

$$
\begin{aligned}
d(g, n, m)= & \frac{n-m}{2}-\sum_{p=0}^{\infty}\left(g^{2}-1\right)^{p}(-1)^{p} \\
& \times \sum_{r=0}^{p}\left(\begin{array}{c}
p \\
r
\end{array}\right) c_{r+1}(1-m, 1-n),
\end{aligned}
$$

while from Eq. (4a) we have

$$
\begin{aligned}
d(g, n, m)= & c_{1}(n, m)+\sum_{p=1}^{\infty}\left(g^{2}-1\right)^{p} \\
& \times\left[c_{p}(n, m)+c_{p+1}(n, m)\right] .
\end{aligned}
$$

We are thus led to the relation

$$
c_{p}(n, m)+c_{p+1}(n, m)
$$

$$
=(-1)^{p+1} \sum_{r=0}^{p}\left(\begin{array}{l}
p \\
r
\end{array}\right) c_{r+1}(1-m, 1-n),
$$

Now we employ the binomial inversion formula ${ }^{22}$

$$
a_{p}=(-1)^{p} \sum_{q=0}^{p}\left(\begin{array}{l}
p \\
q
\end{array}\right) b_{q} \text { for all } p \geqslant 0
$$

$$
\Leftrightarrow \quad b_{p}=(-1)^{p} \sum_{q=0}^{p}\left(\begin{array}{l}
p \\
q
\end{array}\right) a_{q} \text { for all } p \geqslant 0
$$

which is valid for arbitrary $a_{p}$ and $b_{p}$. When applied to Eq. (20) it yields

$$
c_{p}(n, m)=(-1)^{p} \sum_{r=0}^{p}\left(\begin{array}{l}
p \\
r
\end{array}\right) c_{r}(1-m, 1-n) .
$$

We stress that the relations in Eqs. (20) and (22) are valid in arbitrary dimensions for lattices with hopping between $A$ and $B$ sublattices only. In the next section this equation will be used to calculate $c_{p}$ in $D=1$.

\section{Momentum distribution}

An analogous procedure is used to derive relations for $f_{p \sigma}$. We define the abbreviations

$F_{p \sigma}(\boldsymbol{k}, n, m)=f_{p+2 \sigma}(\boldsymbol{k}, n, m)+n_{\boldsymbol{k} \sigma}^{0} f_{p+1 \sigma}(\boldsymbol{k}, n, m)$,

$\bar{F}_{p \uparrow}(\boldsymbol{k}, n, m)=F_{p \uparrow}(\boldsymbol{Q}-\boldsymbol{k}, 1-m, 1-n)$,

$\bar{F}_{p \downarrow}(\boldsymbol{k}, n, m)=F_{p \downarrow}(\boldsymbol{k}, 1-m, 1-n)$,

and rewrite Eq. (4b) as

$$
\begin{aligned}
n_{\boldsymbol{k} \sigma}(g, n, m)= & n_{\boldsymbol{k} \sigma}^{0}(n, m)+\frac{1}{(1+g)^{2}} \\
& \times \sum_{p=0}^{\infty}\left(g^{2}-1\right)^{p+2} F_{p \sigma}(\boldsymbol{k}, n, m) .
\end{aligned}
$$

This expression appears on the left-hand sides of Eqs. (14b) and (14c), while their right-hand sides take the form 


$$
\begin{aligned}
& 1-n_{\boldsymbol{Q}-\boldsymbol{k} \uparrow}\left(g^{-1}, 1-m, 1-n\right) \\
& =n_{\boldsymbol{k} \uparrow}^{0}(n, m)+\frac{1}{(1+g)^{2}} \sum_{p=0}^{\infty} \frac{\left(g^{2}-1\right)^{p+2}}{\left(-g^{2}\right)^{p+1}} \bar{F}_{p \uparrow}(\boldsymbol{k}, n, m), \\
& n_{\boldsymbol{k} \downarrow}\left(g^{-1}, 1-m, 1-n\right) \\
& =n_{\boldsymbol{k} \downarrow}^{0}(n, m)-\frac{1}{(1+g)^{2}} \sum_{p=0}^{\infty} \frac{\left(g^{2}-1\right)^{p+2}}{\left(-g^{2}\right)^{p+1}} \bar{F}_{p \downarrow}(\boldsymbol{k}, n, m) .
\end{aligned}
$$

Expanding $\left(g^{2}\right)^{-p-1}$ in powers of $\left(g^{2}-1\right)$ [Eq. (17)], comparing coefficients, and combining both cases, we find

$$
F_{p \sigma}(\boldsymbol{k}, n, m)=-\operatorname{sgn}(\sigma)(-1)^{p} \sum_{r=0}^{p}\left(\begin{array}{l}
p \\
r
\end{array}\right) \bar{F}_{r \sigma}(\boldsymbol{k}, n, m) .
$$

For clarity we will from now on label $f_{p \sigma}, h_{p \sigma}$, and $n_{k \sigma}$ with the subscripts $<$ and $>$, depending on whether the momentum lies inside or outside of the Fermi surface. We first simplify the equations for $f_{p \uparrow}$. Using Eq. (26) together with Eq. (22) we obtain

$$
h_{p+1 \uparrow}(\boldsymbol{k}, n, m)=(-1)^{p} \sum_{r=1}^{p}\left(\begin{array}{l}
p \\
r
\end{array}\right) h_{r+1 \uparrow}(\boldsymbol{Q}-\boldsymbol{k}, 1-m, 1-n),
$$

valid for all $\boldsymbol{k}$, relating $h_{p \uparrow}^{<}$and $h_{p \uparrow}^{>}$. For the minority spin a similar calculation yields

$$
f_{p \downarrow}^{<}(\boldsymbol{k}, n, m)=(-1)^{p} \sum_{r=0}^{p}\left(\begin{array}{l}
p \\
r
\end{array}\right) f_{r \downarrow}^{<}(\boldsymbol{k}, 1-m, 1-n),
$$

$f_{p+2 \downarrow}^{>}(\boldsymbol{k}, n, m)=(-1)^{p} \sum_{r=0}^{p}\left(\begin{array}{l}p \\ r\end{array}\right) f_{r+2 \downarrow}^{>}(\boldsymbol{k}, 1-m, 1-n)$,

i.e., there is a relation between the $f_{p \downarrow}$ for momenta inside of the Fermi surface, and another relation for momenta outside of the Fermi surface, each linking the cases $n+m \leqslant 1$ and $n+m \geqslant 1$. Note that the relations in Eqs. (27) and (28) are valid in arbitrary dimensions for lattices with hopping between $A$ and $B$ sublattices only.

\section{ANALYTIC EVALUATION IN $D=1$}

In the remainder of the paper we consider the GWF for the Hubbard chain with a symmetric dispersion $\epsilon_{k}=\epsilon_{-k}$ that increases monotonically with $|k|$. This implies that $n_{k \sigma}$ $=n_{-k \sigma}$; therefore we only consider $k \geqslant 0$.

For such dispersions the free Fermi sea described by the starting wave function $\left|\Phi_{0}\right\rangle$ is centered around $k=0$ and is simply connected, i.e., $n_{k \sigma}^{0}=\Theta\left(k_{\mathrm{F} \sigma}-|k|\right)$ is a step function. (We follow the convention of MV of measuring $k$ in units of $2 \pi / a$, where $a$ is the lattice spacing; the first Brillouin zone is the interval $[-1 / 2 ; 1 / 2]$, the reciprocal-lattice vectors $K$ are integers, and the nesting vector $Q=1 / 2$.) For this Fermisurface topology the Fermi momentum, $k_{\mathrm{F} \sigma}=n_{\sigma} / 2=[n$ $+\operatorname{sgn}(\sigma) m] / 4$, only depends on the particle density $n_{\sigma}$, i.e., the particular form of the dispersion is irrelevant. In general this simplification occurs only in dimension $D=1$; higherdimensional tight-binding dispersions usually do not depend only on $|\boldsymbol{k}|$, although this symmetry can be artificially imposed to allow the construction of a dispersion from a given density of states. ${ }^{23}$

Since the values of the diagrams $c_{p}$ and $f_{p \sigma}$ are completely determined by the function $n_{k \sigma}^{0}$ (which is independent of the dispersion as long as $\epsilon_{k}$ is increasing with $|k|$ ) the relations derived in Sec. II B, and hence the relations in Eqs. (20), (22), (27), and (28), are valid for all increasing and symmetric dispersions in $D=1$.

The following analytic calculation of GWF expectation values with magnetization $m \neq 0$ is based on the corresponding calculation for $m=0$ by MV. The calculation for $m=0$ was made possible by exploiting the relations following from canonical transformations, the polynomial form of the diagrams and their continuity as functions of $k$ and $n$, and an analysis of the contribution of umklapp processes. We will now use very similar methods to express the double occupation $d(g, n, m)$ and the momentum distribution $n_{k \sigma}(g, n, m)$ in terms of the known quantities for $m=0$.

In Sec. III A we review the results of MV and present closed formulas for $n_{k \sigma}$ for zero magnetization. For the magnetic case the double occupation, the momentum distribution, and its discontinuity at the Fermi surface are calculated in Secs. III B-D. The variational energy is evaluated in Sec. III E.

\section{A. Zero magnetization}

For the nonmagnetic case the diagrams $c_{p}$ and $h_{p \sigma}$ were already calculated by MV. For $m=0, n \leqslant 1,0 \leqslant k \leqslant 1 / 2$, the results may be summarized as

$$
\begin{gathered}
c_{p}(n, 0)=\gamma_{p} n^{p+1}, \\
f_{p \sigma}(k, n, 0)=h_{p \sigma}(k, n, 0)+c_{p-1}(n, 0)
\end{gathered}
$$




$$
= \begin{cases}n^{p} R_{p}\left(\frac{k}{n}\right) & \text { for } k<\frac{n}{4} \\ n^{p}\left[Q_{p}\left(\frac{k}{n}\right)+\gamma_{p-1}\right] & \text { for } \frac{n}{4}<k \leqslant \min \left(\frac{3 n}{4}, 1-\frac{3 n}{4}\right) \\ n^{p} \gamma_{p-1} & \text { for } \frac{3 n}{4} \leqslant k \leqslant \frac{1}{2} \\ n^{p}\left[Q_{p}\left(\frac{k}{n}\right)+Q_{p}\left(\frac{1-k}{n}\right)+\gamma_{p-1}\right] & \text { for } 1-\frac{3 n}{4} \leqslant k \leqslant \frac{1}{2}\end{cases}
$$

Here and below $\gamma_{p}=(-1)^{p+1} /[2(p+1)]$, and $R_{p}(k)$ and $Q_{p}(k)$ are certain polynomials of degree $p$ in $k$. Note that at $k_{\mathrm{F}}=n / 4$ both $f_{p \sigma}$ and $h_{p \sigma}$ are discontinuous, and $h_{p \sigma}=0$ for $k \geqslant 3 n / 4$. The contribution with momentum $1-k$ in Eq. (31) is due to umklapp processes. From the diagrammatic series in Eq. (4) MV's result for the double occupation is

$d(g, n, 0)=\frac{g^{2}}{2\left(1-g^{2}\right)^{2}}\left\{-\ln \left[1-\left(1-g^{2}\right) n\right]-\left(1-g^{2}\right) n\right\}$,

and the momentum distribution $n_{k \sigma}$ is given by

$n_{k \sigma}^{<}(g, n, 0)$

$$
=1-\frac{1-g}{1+g} \frac{n}{2}+\frac{g^{2}}{(1+g)^{2}}\left\{\mathcal{R}_{0}\left[\frac{4 k}{n},\left(1-g^{2}\right) n\right]-1\right\},
$$

$$
\begin{aligned}
n_{k \sigma}^{>}(g, n, 0)= & -\frac{1-g}{1+g} \frac{n}{2} \\
& +\frac{1}{(1+g)^{2}}\left\{-\frac{1}{2} \ln \left[1-\left(1-g^{2}\right) n\right]\right. \\
& +\mathcal{Q}_{0}\left[\frac{4 k-2 n}{n},\left(1-g^{2}\right) n\right] \\
& \left.+\mathcal{Q}_{0}\left[\frac{4(1-k)-2 n}{n},\left(1-g^{2}\right) n\right]\right\},
\end{aligned}
$$

for $k<k_{\mathrm{F}}$ and $k>k_{\mathrm{F}}$, respectively. Here we introduced the generating functions

$$
\begin{gathered}
\mathcal{R}_{0}(x, z)=\sum_{p=0}^{\infty} R_{p}\left(\frac{x}{4}\right)(-z)^{p}, \\
\mathcal{Q}_{0}(x, z)=\sum_{p=0}^{\infty} Q_{p}\left(\frac{2+x}{4}\right)(-z)^{p},
\end{gathered}
$$

with the convention that $\mathcal{R}_{0}(x, z)$ and $\mathcal{Q}_{0}(x, z)$ are zero for $|x|>1$.

In MV the coefficients of the polynomials $R_{p}(k)$ and $Q_{p}(k)$ had to be calculated recursively to obtain the momen- tum distribution, and convergence was problematic for (1 $\left.-g^{2}\right) n$ close to 1 . In Appendix A we show how the recursion equations for $R_{p}(k)$ can in fact be solved in closed form [Eqs. (A5)-(A8)], while $Q_{p}(k)$ can be expressed in terms of them and their integrals. The generating functions are calculated as

$$
\mathcal{R}_{0}(x, z)=\frac{4 / \pi}{\sqrt{(2-z)^{2}-(x z)^{2}}} \boldsymbol{K}\left(\frac{z \sqrt{1-x^{2}}}{\sqrt{(2-z)^{2}-(x z)^{2}}}\right),
$$

$$
\mathcal{Q}_{0}(x, z)=\mathcal{W}_{0}(x, z)+\frac{z}{2}\left[(1-x) \mathcal{R}_{0}(x, z)+\mathcal{R}_{1}(x, z)\right],
$$

for $|x| \leqslant 1$. Here $\boldsymbol{K}(k)=\int_{0}^{\pi / 2}\left(1-k^{2} \sin ^{2} \phi\right)^{-1 / 2} d \phi$ is the complete elliptic integral of the first kind, $\mathcal{W}_{0}(x, z)$ is an auxiliary function,

$$
\begin{aligned}
\mathcal{W}_{0}(x, z)= & \frac{x-1}{2} \mathcal{R}_{0}(x, z)+\frac{z-2}{4} \mathcal{R}_{1}(x, z) \\
& +\frac{z(z-1)}{2} \dot{\mathcal{R}}_{1}(x, z),
\end{aligned}
$$

where the dot indicates a partial derivative with respect to second argument, and $\mathcal{R}_{j}(x, z)$ is the repeated integral of $\mathcal{R}_{0}(x, z)$, defined by $(j \geqslant 0)$

$$
\begin{aligned}
\mathcal{R}_{j+1}(x, z) & =\int_{1}^{x} d x^{\prime} \mathcal{R}_{j}\left(x^{\prime}, z\right) \\
& =\frac{1}{j !} \int_{1}^{x} d x^{\prime}\left(x-x^{\prime}\right)^{j} \mathcal{R}_{0}\left(x^{\prime}, z\right)
\end{aligned}
$$

Below we will also need the following integral,

$$
\begin{aligned}
\mathcal{Q}_{1}(x, z)= & \int_{1}^{x} d x^{\prime} \mathcal{Q}_{0}\left(x^{\prime}, z\right) \\
= & \frac{(1-z)(x-1)}{2} \mathcal{R}_{1}(x, z)+\frac{5 z-4}{4} \mathcal{R}_{2}(x, z) \\
& +\frac{z(z-1)}{2} \dot{\mathcal{R}}_{2}(x, z) .
\end{aligned}
$$


In Appendix A we provide an explicit expression for $\mathcal{R}_{j}(x, z)$ [Eqs. (A10) and (A11)], as well as other relations. Here we note in particular the following functional relations, which are obtained from Eqs. (37) and (38),

$$
\begin{aligned}
& \mathcal{R}_{j}\left(x, \frac{z}{z-1}\right)=(1-z) \mathcal{R}_{j}(x, z), \\
& \mathcal{Q}_{j}\left(x, \frac{z}{z-1}\right)=\mathcal{W}_{j}(x, z) .
\end{aligned}
$$

These expressions analytically continue $\mathcal{R}_{j}(x, z)$ and $\mathcal{Q}_{j}(x, z)$ to $|z|>1$.

\section{B. Nonzero magnetization: Double occupation}

To calculate the double occupation one needs the diagrams $c_{p}$. Using the methods of MV one can show that for $m>0$ umklapp processes occur in the graphs for $c_{p}(n, m)$ only if $n \geqslant 1$. This implies that for $n<1 c_{p}(n, m)$ is a homogeneous function of $k_{\mathrm{F} \uparrow}$ and $k_{\mathrm{F} \downarrow}$ of order $p+1$, since every contributing graph contains $p+1$ free momenta. Since $k_{\mathrm{F} \sigma}$ $=[n+\operatorname{sgn}(\sigma) m] / 4$ it follows that $c_{p}(n, m)$ can be written as a linear combination of terms $n_{\uparrow}^{r} n^{p+1-r}$ with $0 \leqslant r \leqslant p+1$, i.e., $c_{p}(n, m) / n^{p+1}$ is a polynomial in $m / n$ of degree $p+1$. This is also the case for $n=1$ since $d(g, n, m)$ is continuous at $n=1$ [see Eq. (11a)]. Therefore we can write the polynomial simply as $c_{p}(n, m) / n^{p+1}=c_{p}(1, m / n)$ for $n \leqslant 1$. For $n$ $=1$, however, we can calculate $c_{p}(1, m)$ from Eqs. (22) and (29):

$c_{p}(1, m)=(-1)^{p} \sum_{r=0}^{p}\left(\begin{array}{l}p \\ r\end{array}\right) c_{r}(1-m, 0)=\gamma_{p}\left(1-m^{p+1}\right)$.

The polynomial structure of $c_{p}(n, m)$ then implies

$$
\begin{aligned}
c_{p}(n, m) & =n^{p+1} c_{p}\left(1, \frac{m}{n}\right)=\gamma_{p}\left(n^{p+1}-m^{p+1}\right), \\
& =c_{p}(n, 0)-c_{p}(m, 0) .
\end{aligned}
$$

Summation of the series in Eq. (4a) yields the simple result

$$
d(g, n, m)=d(g, n, 0)-d(g, m, 0) .
$$

It is remarkable that the double occupation at density $n$ and magnetization $m$ is obtained as the difference between the double occupation without magnetization at density $n$ and density $m$. With the closed form of $d(g, n, 0)$ taken from Eq. (32) we finally obtain for the double occupation, valid for $0 \leqslant m \leqslant n \leqslant 1$,

$d(g, n, m)$

$$
=\frac{g^{2}}{2\left(1-g^{2}\right)^{2}}\left(\ln \frac{1-\left(1-g^{2}\right) m}{1-\left(1-g^{2}\right) n}-\left(1-g^{2}\right)(n-m)\right),
$$

The double occupation is shown in Fig. 1 for various parameter values. In the limit of strong correlation $(g \rightarrow 0)$ it behaves as

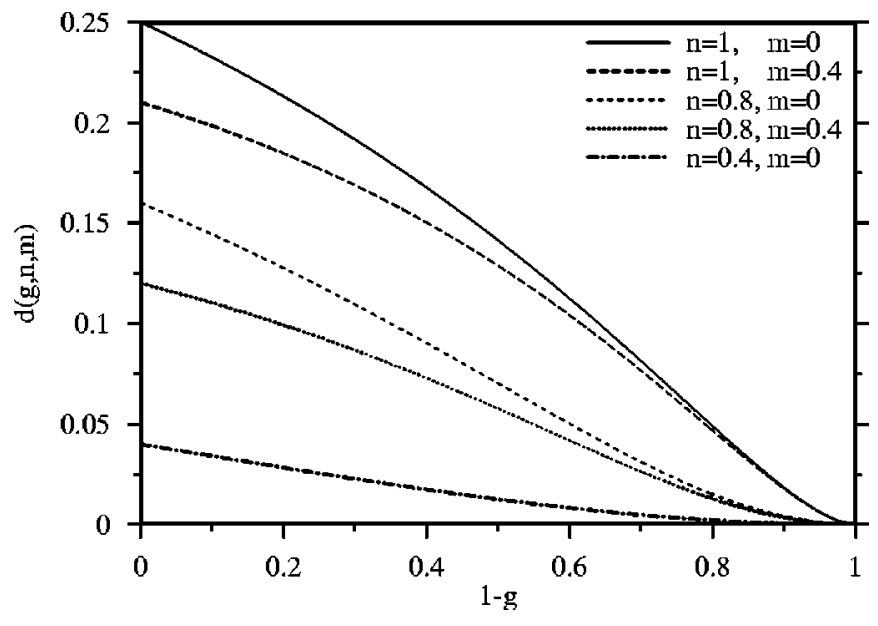

FIG. 1. Double occupation $d(g, n, m)$ as a function of the variational parameter $g$ for various densities $n$ and magnetizations $m$. Note that $d(g, n, m)=d(g, n, 0)-d(g, m, 0)$.

$d(g, n<1, m \leqslant n)=\frac{g^{2}}{2}\left[\ln \frac{1-m}{1-n}-(n-m)\right]+O\left(g^{4}\right)$,

$d(g, n=1, m<1)$

$$
=g^{2}\left[\ln \frac{1}{g}+\frac{\ln (1-m)-(1-m)}{2}\right]+O\left(g^{4} \ln g\right),
$$

i.e., the double occupation is nonanalytic in the limit $n$ $\rightarrow 1, g \rightarrow 0$, as in the nonmagnetic case (see MV).

\section{Nonzero magnetization: Momentum distribution}

To calculate the momentum distribution $n_{k \sigma}$ one needs the diagrams $f_{p \sigma}$ or $h_{p \sigma}$. In the following subsections we distinguish the cases of majority and minority spin, as well as whether $k$ lies inside or outside of the Fermi surface.

For finite magnetization, $m>0$, we make the following observations, similar in spirit to those of MV for the nonmagnetic case. As a function of $k, f_{p \sigma}(k, n, m)$ and $h_{p \sigma}(k, n, m)$ are discontinuous at $k=k_{\mathrm{F} \sigma}$, since the oneparticle irreducible graphs contain a factor $n_{k \sigma}^{0}$. For $k \geqslant k_{\mathrm{F} \sigma}$ $+2 k_{\mathrm{F}-\sigma}$ momentum conservation at the outer vertices of $h_{p \sigma}$ cannot be fulfilled, so that in this case $h_{p \sigma}(k, n, m)=0$. For $k \geqslant 1-k_{\mathrm{F} \sigma}-2 k_{\mathrm{F}-\sigma}\left(\geqslant k_{\mathrm{F} \sigma}\right)$ umklapp processes occur and yield an additional contribution to $h_{p \sigma}$ of normal processes with external momentum $1-k$. In the absence of umklapp processes $f_{p \sigma}$ and $h_{p \sigma}$ are homogeneous functions of $k_{\mathrm{F} \uparrow}$ and $k_{\mathrm{F} \downarrow}$ of order $p$, since every contributing graph contains $p$ free momenta. Then $f_{p \sigma} / n^{p}$ and $h_{p \sigma} / n^{p}$ are polynomials in $k / n$ and $m / n$ of degree $p$, and due to momentum conservation at outer vertices different polynomials occur depending on whether $k$ is larger or smaller than $\pm\left(2 k_{\mathrm{F}-\sigma}\right.$ $\left.-k_{\mathrm{F} \sigma}\right)$. Furthermore, different polynomials for $h_{p \downarrow}^{>} / n^{p}$ occur also depending on how $k$ compares to $3 k_{\mathrm{F} \downarrow}$ (see Sec. III C 4 below and Appendix B).

\section{Majority spins inside of the Fermi surface: $n_{k \uparrow}^{<}(g, n, m)$}

For $0 \leqslant k<k_{\mathrm{F} \uparrow}=(n+m) / 4$ and $n=1$ the diagrams $h_{p \uparrow}^{<}$can be obtained from Eq. (27) in terms of the known functions $h_{p \uparrow}^{>}$of the nonmagnetic case [Eq. (31)]: 


$$
\begin{aligned}
& h_{p+1 \uparrow}^{<}(k, 1, m)=(-1)^{p} \sum_{r=1}^{p}\left(\begin{array}{l}
p \\
r
\end{array}\right) h_{r+1 \uparrow}^{>}\left(\frac{1}{2}-k, 1-m, 0\right) \\
& =(-1)^{p} \sum_{r=1}^{p}\left(\begin{array}{l}
p \\
r
\end{array}\right)(1-m)^{r+1}\left\{\begin{array}{ll}
Q_{r+1}\left(\frac{\frac{1}{2}-k}{1-m}\right) & \text { for } \frac{|3 m-1|}{4} \leqslant k<\frac{1+m}{4} \\
0 & \text { for } 0 \leqslant k \leqslant \frac{3 m-1}{4} \\
{\left[Q_{r+1}\left(\frac{\frac{1}{2}-k}{1-m}\right)+Q_{r+1}\left(\frac{\frac{1}{2}+k}{1-m}\right)\right]} & \text { for } 0 \leqslant k \leqslant \frac{1-3 m}{4}
\end{array} .\right.
\end{aligned}
$$

Since umklapp processes do not occur for $k<k_{\mathrm{F} \uparrow}, h_{p \uparrow}^{<}(k, n, m) / n^{p}$ is a polynomial in $k / n$ and $m / n$. Hence we can obtain $h_{p+1 \uparrow}^{<} / n^{p+1}$ by replacing $k$ by $k / n$ and $m$ by $m / n$ in Eq. (50). This yields

$$
h_{p+1 \uparrow}^{<}(k, n, m)=(-1)^{p} \sum_{r=1}^{p}\left(\begin{array}{l}
p \\
r
\end{array}\right)(n-m)^{r+1} n^{p-r}\left\{\begin{array}{ll}
Q_{r+1}\left(\frac{\frac{n}{2}-k}{n-m}\right) & \text { for } \frac{|3 m-n|}{4} \leqslant k<\frac{n+m}{4} \\
0 & \text { for } 0 \leqslant k \leqslant \frac{3 m-n}{4} \\
{\left[Q_{r+1}\left(\frac{\frac{n}{2}-k}{n-m}\right)+Q_{r+1}\left(\frac{\frac{n}{2}+k}{n-m}\right)\right]} & \text { for } 0 \leqslant k \leqslant \frac{n-3 m}{4}
\end{array} .\right.
$$

Note that $h_{p+1 \uparrow}^{<}(k, n, m)$ is continuous for all $k<k_{\mathrm{F} \uparrow}$ due to $Q_{p}(3 / 4)=0$ (see MV). The normal processes contribute differently depending on how $k$ compares to $\pm\left(2 k_{\mathrm{F} \downarrow}-k_{\mathrm{F} \uparrow}\right)$, as expected.

Since $h_{p \uparrow}^{<}$is available for arbitrary orders of $p$, the series in Eq. (4b) can now be summed, with the result, valid for 0 $\leqslant m<n \leqslant 1,0 \leqslant k<(n+m) / 4$,

$$
\begin{aligned}
n_{k \uparrow}^{<}(g, n, m) & =1-(1-g)^{2}\left[\frac{n-m}{2}-d(g, n, m)\right]-\frac{g^{2}}{(1+g)^{2}}\left[\mathcal{W}_{0}\left(\frac{2 m-4 k}{n-m}, \frac{\left(1-g^{2}\right)(n-m)}{1-\left(1-g^{2}\right) m}\right)\right. \\
& \left.+\mathcal{W}_{0}\left(\frac{2 m+4 k}{n-m}, \frac{\left(1-g^{2}\right)(n-m)}{1-\left(1-g^{2}\right) m}\right)\right],
\end{aligned}
$$

where the functional relation in Eq. (43) was used. For $m=0$ this result reduces to Eq. (34) by virtue of Eq. (A12).

\section{Majority spins outside of the Fermi surface: $n_{k \uparrow}^{>}(g, n, m)$}

For $(n+m) / 4<k \leqslant 1 / 2$ we can deduce $h_{p \uparrow}^{>}$at once by inserting $h_{p \uparrow}^{<}$into Eq. (27):

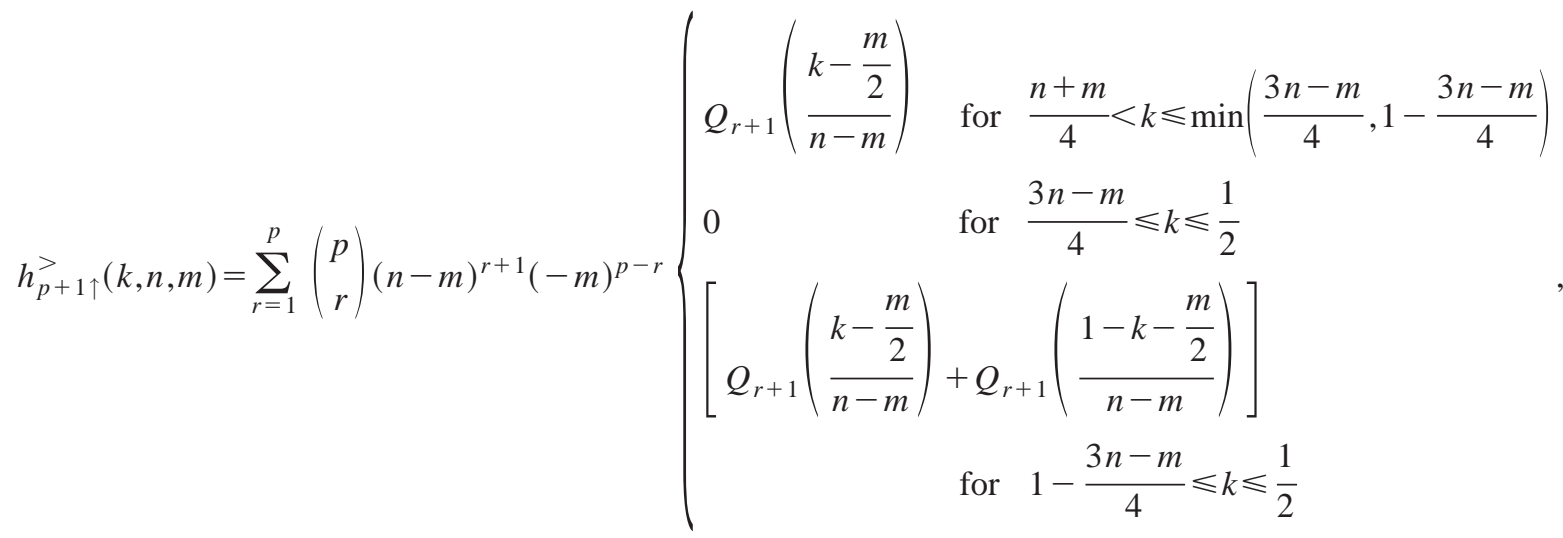



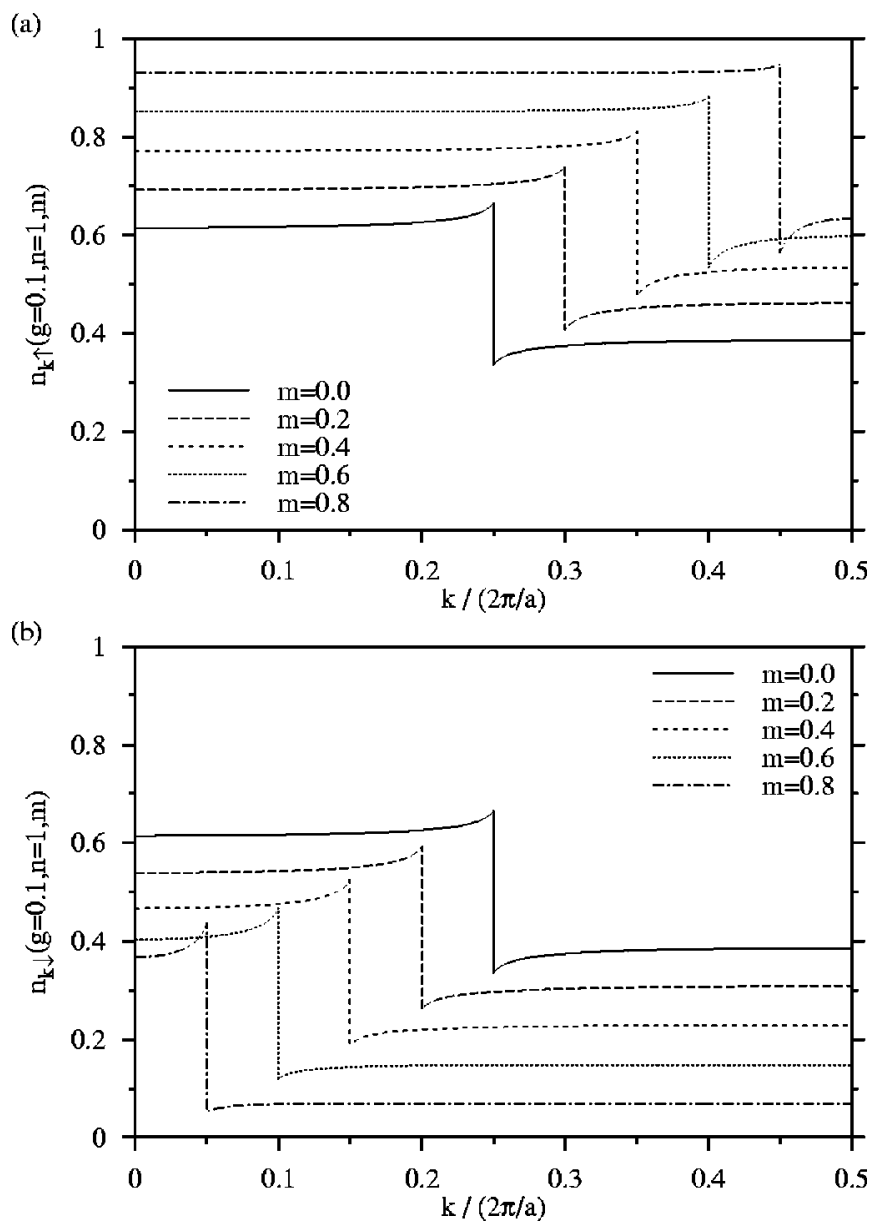

FIG. 2. Momentum distribution $n_{k \sigma}$ of (a) majority and (b) minority spin electrons, as a function of $k$ for $g=0.1$ and density $n=1$.

which is continuous for all $k>k_{\mathrm{F} \uparrow}$. In this sector umklapp processes occur for $k \geqslant 1-2 k_{\mathrm{F} \downarrow}-k_{\mathrm{F} \uparrow}$ and contribute to $h_{p \uparrow}^{<}$like normal processes with external momentum $1-k$.

Summing the series in Eq. (4b) then yields the result, valid for $0 \leqslant m<n \leqslant 1,(n+m) / 4<k \leqslant 1 / 2$,

$$
\begin{aligned}
n_{k \uparrow}^{>}(g, n, m)= & \frac{(1-g)^{2}}{g^{2}} d(g, n, m) \\
& +\frac{1}{(1+g)^{2}}\left[\mathcal{Q}_{0}\left(\frac{4 k-2 n}{n-m}, \frac{\left(1-g^{2}\right)(n-m)}{1-\left(1-g^{2}\right) m}\right)\right. \\
& \left.+\mathcal{Q}_{0}\left(\frac{4(1-k)-2 n}{n-m}, \frac{\left(1-g^{2}\right)(n-m)}{1-\left(1-g^{2}\right) m}\right)\right],
\end{aligned}
$$

which alternatively can be derived directly from Eqs. (14b), (52), and (43). Note also that for $m=0$ this result reduces to Eq. (34). The momentum distribution $n_{k \uparrow}(g, n, m)$
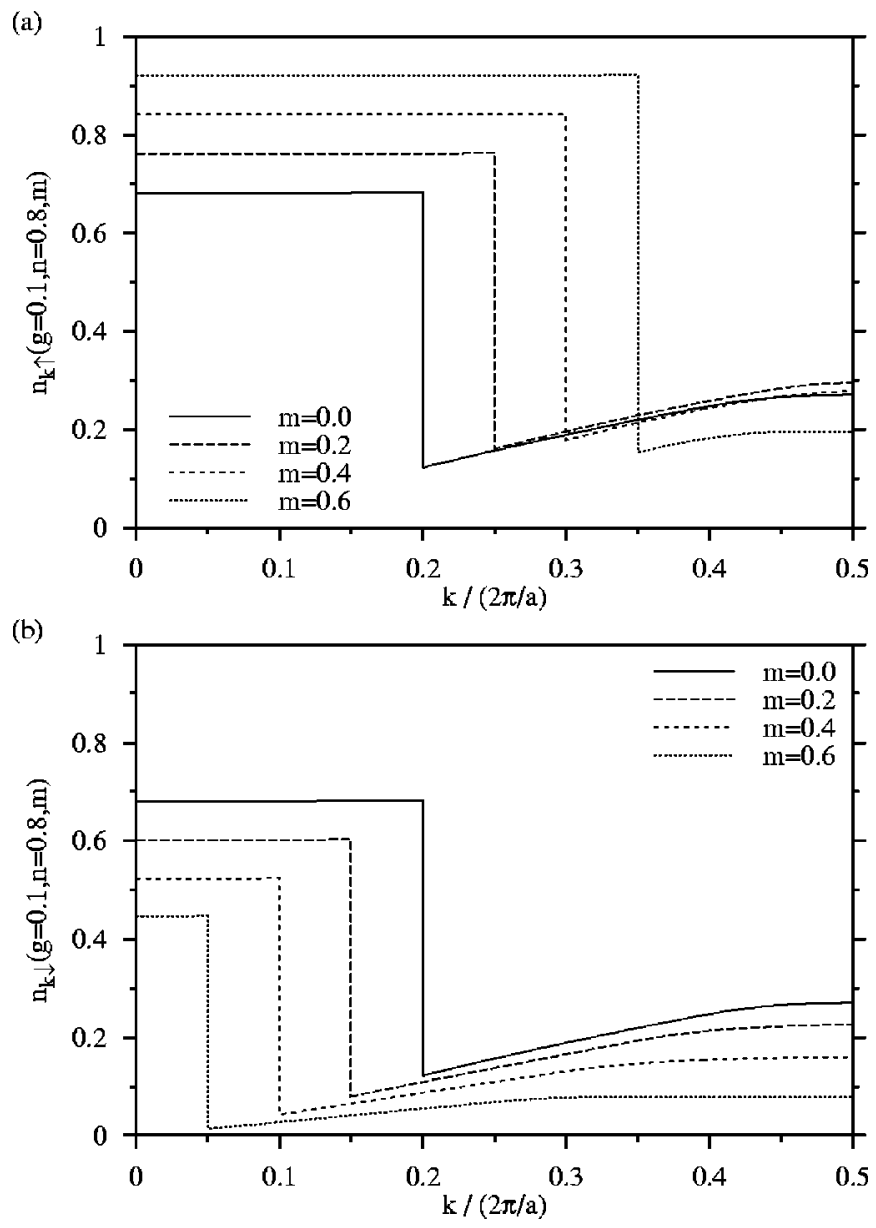

FIG. 3. Momentum distribution $n_{k \sigma}$ of (a) majority and (b) minority spin electrons as a function of $k$ for $g=0.1$ and density $n$ $=0.8$.

has thus been determined in the entire parameter range. It is shown for $g=0.1$ and densities $n=1$ and 0.8 in Figs. 2(a) and $3(\mathrm{a})$.

\section{Minority spins inside of the Fermi surface: $n_{k \downarrow}^{<}(g, n, m)$}

For $0 \leqslant k<k_{\mathrm{F} \downarrow}=(n-m) / 4$ and $n=1$ the diagrams $f_{p \downarrow}^{<}$are given in terms of the known functions $f_{p \uparrow}^{<}$of the nonmagnetic case [Eq. (31)] according to Eq. (28a):

$$
f_{p \downarrow}^{<}(k, 1, m)=(-1)^{p} \sum_{r=0}^{p}\left(\begin{array}{l}
p \\
r
\end{array}\right)(1-m)^{r} R_{r}\left(\frac{k}{1-m}\right) .
$$

Due to the absence of umklapp processes for $k<k_{\mathrm{F} \downarrow}$, $f_{p \downarrow}^{<}(k, n, m) / n^{p}$ is a polynomial in $k / n$ and $m / n$. Therefore it is given by Eq. (55) with $k$ replaced by $k / n$ and $m$ replaced by $m / n$. We then have

$$
\begin{aligned}
f_{p \downarrow}^{<}(k, n, m) & =(-1)^{p} \sum_{r=0}^{p}\left(\begin{array}{c}
p \\
r
\end{array}\right)(n-m)^{r} n^{p-r} R_{r}\left(\frac{k}{n-m}\right), \\
& =\sum_{r=0}^{p}\left(\begin{array}{l}
p \\
r
\end{array}\right)(n-m)^{r}(-m)^{p-r} R_{r}\left(\frac{k}{n-m}\right)
\end{aligned}
$$


where the second equation was obtained by inserting Eq. (56) in Eq. (28a); it is equivalent to the functional relation in Eq. (42). Note that for $m=0 \mathrm{Eq}$. (57) reduces to Eq. (31).

We use Eq. (57) in Eq. (4b) to find the following expression for $n_{k \downarrow}^{<}$, valid for $0 \leqslant m<n \leqslant 1, \quad 0 \leqslant k<(n$ $-m) / 4$,

$$
\begin{aligned}
n_{k \downarrow}^{<}(g, n, m)=1 & -\frac{1-g}{1+g} \frac{n+m}{2}+\frac{g^{2}}{(1+g)^{2}} \\
& \times\left[\frac{\mathcal{R}_{0}\left(\frac{4 k}{n-m}, \frac{\left(1-g^{2}\right)(n-m)}{1-\left(1-g^{2}\right) m}\right)}{1-\left(1-g^{2}\right) m}-1\right] .
\end{aligned}
$$

This expression reduces to Eq. (33) for $m=0$.

\section{Minority spins outside of the Fermi surface: $n_{k \downarrow}^{>}(g, n, m)$}

Finally we consider the case $(n-m) / 4<k \leqslant 1 / 2$, for which the calculation of $n_{k \downarrow}^{>}$is somewhat more complicated. We begin with the special case $n=1$, for which the momentum distribution can be determined immediately from Eqs. (14c), (10b), and (14b), which combine to give

$$
\begin{aligned}
n_{k \downarrow}^{>}(g, 1, m) & =n_{k \downarrow}^{>}\left(g^{-1}, 1-m, 0\right) \\
& =n_{k \uparrow}^{>}\left(g^{-1}, 1-m, 0\right)=1-n_{1 / 2-k \uparrow}^{<}(g, 1, m) .
\end{aligned}
$$

Inserting Eq. (52) we arrive at

$$
\begin{aligned}
n_{k \downarrow}^{>}(g, 1, m)= & (1-g)^{2}\left(\frac{1-m}{2}-d(g, 1, m)\right) \\
& +\frac{g^{2}}{(1+g)^{2}}\left[\mathcal{W}_{0}\left(\frac{2 m+4 k-2}{1-m}, \frac{\left(1-g^{2}\right)(1-m)}{1-\left(1-g^{2}\right) m}\right)\right. \\
& \left.+\mathcal{W}_{0}\left(\frac{2 m-4 k+2}{1-m}, \frac{\left(1-g^{2}\right)(1-m)}{1-\left(1-g^{2}\right) m}\right)\right] .
\end{aligned}
$$

Next we consider arbitrary density and magnetization, 0 $\leqslant m<n \leqslant 1$. We make use of the following relation, which follows from Eqs. (28b) and (20),

$$
\begin{aligned}
h_{p+2 \downarrow}^{>}(k, n, m)= & -2 c_{p+1}(n, m)-c_{p}(n, m) \\
& +(-1)^{p} \sum_{r=0}^{p}\left(\begin{array}{c}
p \\
r
\end{array}\right) h_{r+2 \downarrow}^{>}(k, 1-m, 1-n) .
\end{aligned}
$$

The diagrams $h_{p \downarrow}^{>}$that appear in this equation can be written in terms of the contribution of normal processes, $N_{p}$, as

$$
h_{p \downarrow}^{>}(k, n, m)=\left\{\begin{array}{ll}
n^{p} N_{p}\left(\frac{k}{n}, \frac{m}{n}\right) & \text { for } \frac{n-m}{4}<k \leqslant \min \left(\frac{3 n+m}{4}, 1-\frac{3 n+m}{4}\right) \\
0 & \text { for } \frac{3 n+m}{4} \leqslant k \leqslant \frac{1}{2} \\
n^{p}\left[N_{p}\left(\frac{k}{n}, \frac{m}{n}\right)+N_{p}\left(\frac{1-k}{n}, \frac{m}{n}\right)\right] & \text { for } 1-\frac{3 n+m}{4} \leqslant k \leqslant \frac{1}{2}
\end{array} .\right.
$$

In Appendix B we show that depending on $k$ and $m$, the function $N_{p}(k, m)$ is given piecewise by four polynomials in $k$ and $m$ of order $p$. The explicit determination of these polynomials is quite involved; it is presented in Appendix C. The final result for $n_{k \downarrow}^{>}$, valid for $0 \leqslant m<n \leqslant 1,(n-m) / 4<k \leqslant 1 / 2$, can be written as

$$
n_{k \downarrow}^{>}(g, n, m)=\frac{(1-g)^{2}}{g^{2}} d(g, n, m)+\frac{1}{(1+g)^{2}}[N(g, k, n, m)+N(g, 1-k, n, m)],
$$

where $N(g, k, n, m)$ is given by 


$$
N(g, k, n, m)=\left\{\begin{array}{ll}
N^{(1)}(g, k, n, m) & \text { for } \frac{n-m}{4}<k \leqslant \min \left(\frac{n+3 m}{4}, \frac{3(n-m)}{4}\right) \\
N^{(2)}(g, k, n, m) & \text { for } m \leqslant \frac{n}{3} \text { and } \frac{n+3 m}{4} \leqslant k \leqslant \frac{3(n-m)}{4} \\
N^{(3)}(g, k, n, m) & \text { for } m \geqslant \frac{n}{3} \text { and } \frac{3(n-m)}{4} \leqslant k \leqslant \frac{n+3 m}{4} \\
N^{(4)}(g, k, n, m) & \text { for } \max \left(\frac{n+3 m}{4}, \frac{3(n-m)}{4}\right) \leqslant k \leqslant \frac{3 n+m}{4} \\
0 & \text { for } \frac{3 n+m}{4} \leqslant k
\end{array},\right.
$$

with

$$
\begin{aligned}
& N^{(1)}(g, k, n, m)=\frac{\left[1-\left(1-g^{2}\right) m\right](3 n-3 m-4 k)}{2(n-m)} \mathcal{Q}_{0}\left(\frac{4 k-2 n+2 m}{n-m}, \frac{\left(1-g^{2}\right)(n-m)}{1-\left(1-g^{2}\right) m}\right) \\
& +\frac{(n-3 m)\left(1-g^{2}\right)+2}{4} \mathcal{Q}_{1}\left(\frac{4 k-2 n+2 m}{n-m}, \frac{\left(1-g^{2}\right)(n-m)}{1-\left(1-g^{2}\right) m}\right) \\
& +\frac{(n-m)\left(1-g^{2}\right)\left[1-\left(1-g^{2}\right) n\right]}{2\left(1-\left(1-g^{2}\right) m\right)} \dot{\mathcal{Q}}_{1}\left(\frac{4 k-2 n+2 m}{n-m}, \frac{\left(1-g^{2}\right)(n-m)}{1-\left(1-g^{2}\right) m}\right) \\
& +N^{(3)}(g, k, n, m) \text {, } \\
& N^{(4)}(g, k, n, m)=\frac{\left[1-\left(1-g^{2}\right) m\right](4 k-n-3 m)}{2(n-m)} \mathcal{Q}_{0}\left(\frac{4 k-2 n-2 m}{n-m}, \frac{\left(1-g^{2}\right)(n-m)}{1-\left(1-g^{2}\right) m}\right) \\
& -\frac{(n-3 m)\left(1-g^{2}\right)+2}{4} \mathcal{Q}_{1}\left(\frac{4 k-2 n-2 m}{n-m}, \frac{\left(1-g^{2}\right)(n-m)}{1-\left(1-g^{2}\right) m}\right) \\
& -\frac{(n-m)\left(1-g^{2}\right)\left[1-\left(1-g^{2}\right) n\right]}{2\left[1-\left(1-g^{2}\right) m\right]} \dot{\mathcal{Q}}_{1}\left(\frac{4 k-2 n-2 m}{n-m}, \frac{\left(1-g^{2}\right)(n-m)}{1-\left(1-g^{2}\right) m}\right) \text {, }
\end{aligned}
$$

$$
\begin{aligned}
N^{(3)}(g, k, n, m)= & \frac{(n-m)\left(1-g^{2}\right)}{2} \\
& -\frac{1-\left(1-g^{2}\right) k}{2} \ln \frac{1-\left(1-g^{2}\right) m}{1-\left(1-g^{2}\right) n},
\end{aligned}
$$

$$
\begin{aligned}
N^{(2)}(g, k, n, m)= & N^{(1)}(g, k, n, m) \\
& -N^{(3)}(g, k, n, m)+N^{(4)}(g, k, n, m),
\end{aligned}
$$

and the dot again denotes derivative with respect to second argument. It can be checked that Eq. (63) indeed reduces to
Eq. (60) for $m=0$. Thus the momentum distribution $n_{k \downarrow}(g, n, m)$ has been determined for all parameters. It is shown for $g=0.1$ and densities $n=1$ and 0.8 in Figs. 2(b) and $3(\mathrm{~b})$.

The calculation of the correlated momentum distributions $n_{k \sigma}$ is now complete. We remark that they are continuous functions of $k$, except at $k_{\mathrm{F} \sigma}$, and are also continuous in $n$ and $m$ for fixed $k$. We have checked that they obey the sum rule in Eq. (8).

\section{Discontinuity of the momentum distribution at the Fermi surface}

It suffices to calculate the discontinuity $q_{\sigma}$ of $n_{k \sigma}$ at the Fermi surface for $0 \leqslant m<n \leqslant 1$ [see Eq. (12)]. From our pre- 

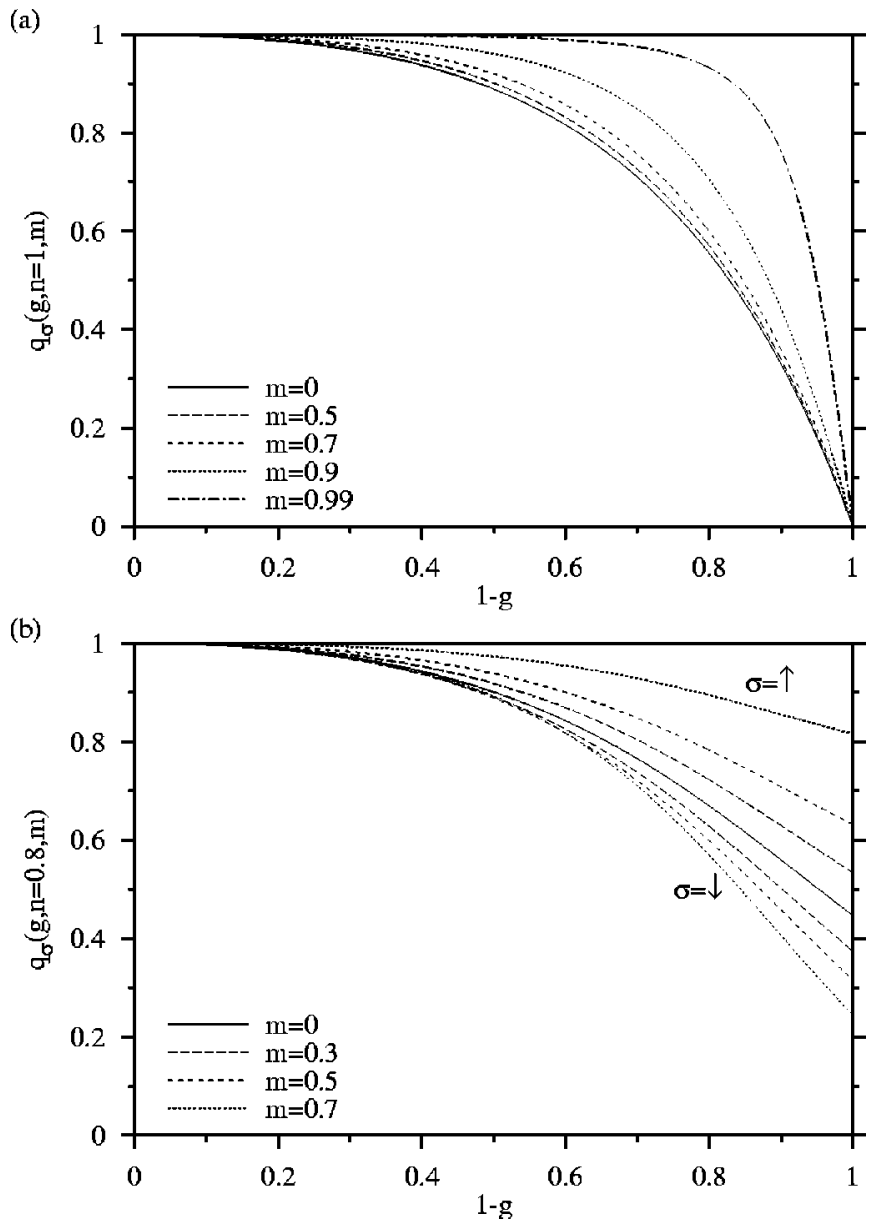

FIG. 4. Discontinuity $q_{\sigma}$ of the momentum distribution at the Fermi vector for densities (a) $n=1$ (in this case $q_{\uparrow}=q_{\downarrow}$ ) and (b) $n=0.8$.

vious results we obtain the left and right limit of the momentum distribution at the Fermi vector as

$$
\begin{gathered}
n_{k_{\mathrm{F} \sigma}^{-} \sigma}(g, n, m)=1-\frac{1-g}{1+g} n_{-\sigma}+\frac{g^{2}\left(G_{\sigma}^{-1}-1\right)}{(1+g)^{2}}, \\
n_{k_{\mathrm{F} \sigma}^{+} \sigma}(g, n, m)=-\frac{1-g}{1+g} n_{-\sigma}+\frac{1-G_{\sigma}}{(1+g)^{2}},
\end{gathered}
$$

where the abbreviation $G_{\sigma}$ is defined as

$$
G_{\sigma}=\sqrt{\left[1-\left(1-g^{2}\right) n\right]\left[1-\left(1-g^{2}\right) m\right]^{-\operatorname{sgn}(\sigma)}} .
$$

For the discontinuity at the $\sigma$-spin Fermi surface we thus obtain

$$
q_{\sigma}(g, n, m)=\frac{\left(g+G_{\sigma}\right)^{2}}{(1+g)^{2} G_{\sigma}} .
$$

It follows that $q_{\sigma}$ vanishes only for a half filled band without double occupation ( $n=1$ and $g=0)$; in this case there is exactly one particle at each site so that $n_{k \sigma}=1 / 2$ for all $k$. Note also that $q_{\uparrow}=q_{\downarrow}$ if $n=1$ (or, trivially, if $m=0$ or $g$ $=1)$. We plot $q_{\sigma}$ for $n=1$ and $n=0.8$ in Fig. 4 .
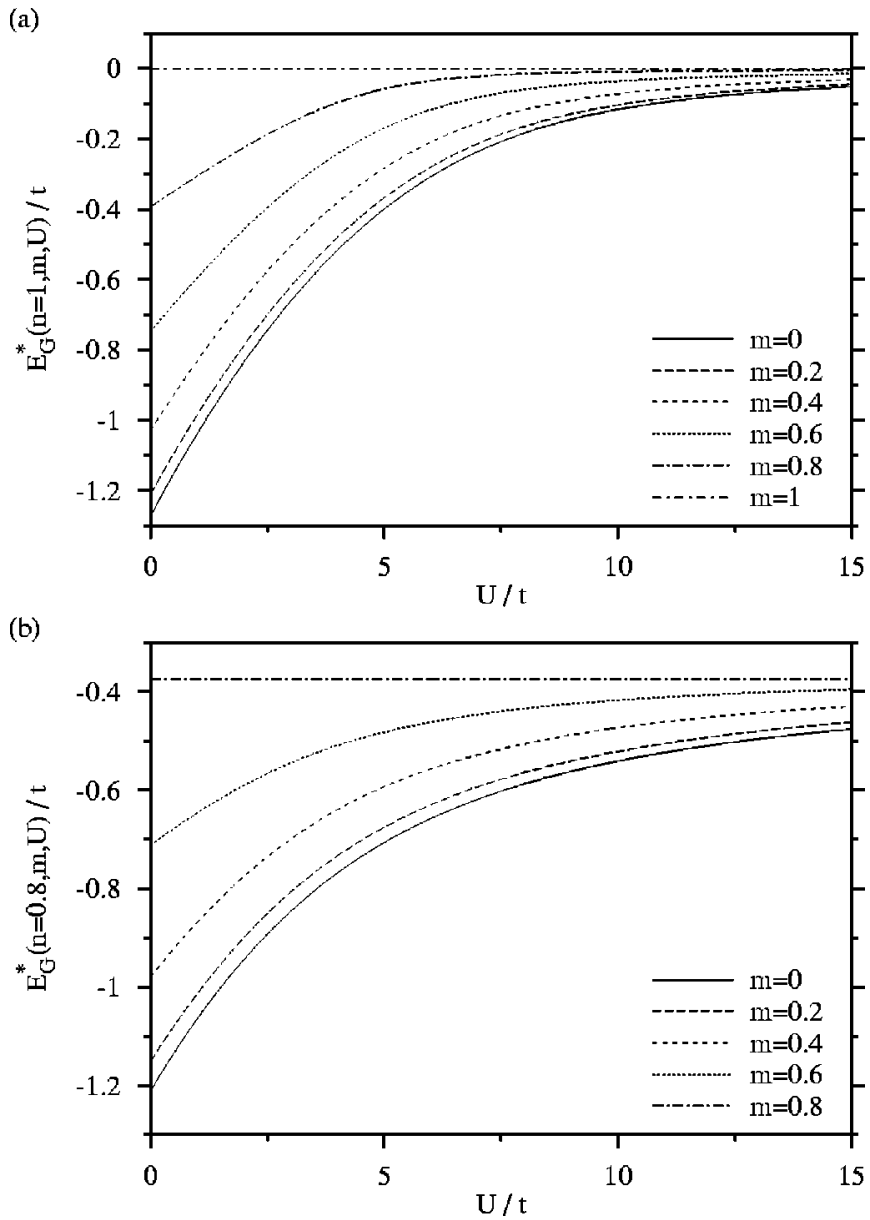

FIG. 5. Energy expectation value for the Hubbard chain with nearest-neighbor hopping $t>0$ for densities (a) $n=1$ and (b) $n=0.8$.

\section{E. Energy expectation value}

For any symmetric dispersion $\epsilon_{k}$, monotonically increasing with $|k|$, we can now calculate the energy expectation value per site, $E_{\mathrm{G}}=\langle\hat{H}\rangle_{\mathrm{G}} / L$, of the one-dimensional Hubbard Hamiltionian (1), which is then minimized with respect to $g$ to find the optimal variational energy, $E_{\mathrm{G}}^{\star}$,

$$
E_{\mathrm{G}}^{\star}(n, m, U)=\min _{0 \leqslant g \leqslant 1} E_{\mathrm{G}}(g, n, m, U),
$$

$$
E_{\mathrm{G}}(g, n, m, U)=2 \int_{0}^{1 / 2} d k \epsilon_{k} \sum_{\sigma} n_{k \sigma}(g, n, m)+U d(g, n, m) .
$$

Note that it follows from Eq. (11b) that the total kinetic energy for dispersion $\epsilon_{k}$ at density $n>1$ can be calculated from the dispersion $-\epsilon_{1 / 2-k}$ at density $2-n$ using the formulas for $n_{k \sigma}$ and $d$ that are valid below half filling.

For the Hubbard chain with nearest-neighbor hopping $t$ the dispersion relation in our notation is $\epsilon_{k}=-2 t \cos (2 \pi k)$. We assume $t>0$ without loss of generality, so that the dispersion is increasing with $|k|$ and our results for the Gutzwiller expectation values apply. The optimal variational energy for this system is shown in Fig. 5 for densities $n$ $=1$ and 0.8 for various magnetizations. Note that at half filling no Brinkman-Rice metal-insulator transition occurs at 
(a)

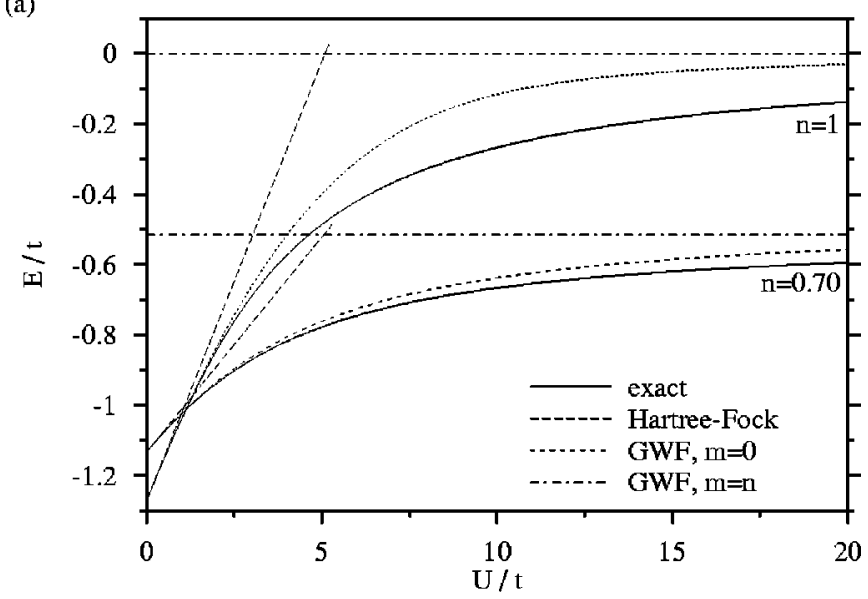

(b)

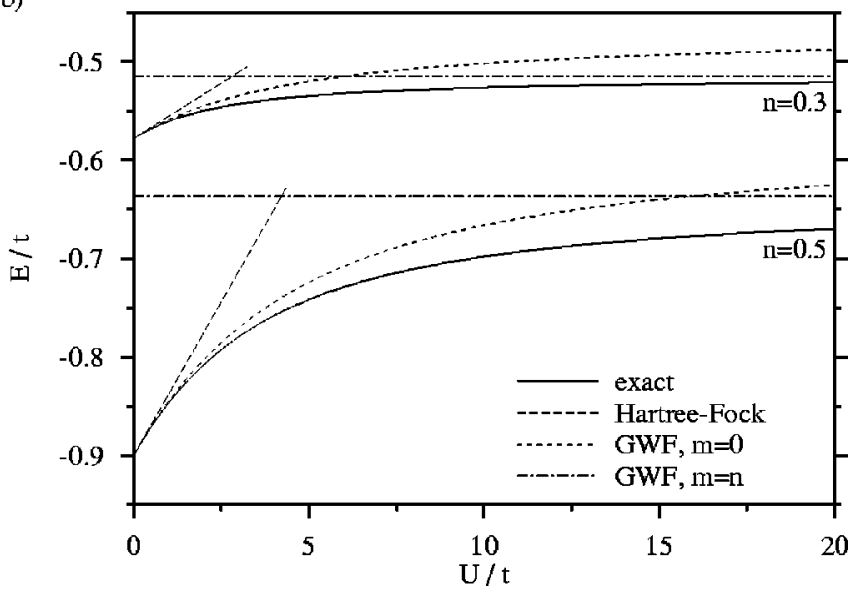

FIG. 6. Comparison of the total energy for the Hubbard chain with nearest-neighbor hopping and for densities (a) $n=0.7,1$ and (b) $n=0.3,0.5$.

any finite $U$; i.e., $g=0$ is the optimal variational parameter only for $U=\infty$. The variational result for the ground-state magnetization is determined in the next section.

\section{MAGNETIC PHASE DIAGRAM OF THE HUBBARD CHAIN}

In this section we determine the instability towards ferromagnetism for the Hubbard chain with nearest-neighbor hopping. Currently only homogeneous paramagnetic and ferromagnetic phases can be investigated analytically with the GWF in $D=1$; hence we do not consider antiferromagnetism or other broken symmetries. We begin by examining the energy for the special cases of the paramagnetic state (i.e., zero magnetization) and the fully polarized state. The latter contains the minimum number of doubly occupied sites and is an eigenstate of $\hat{H}$, with eigenvalue

$$
\begin{aligned}
E_{\mathrm{FP}}(n, U) & =E_{\mathrm{G}}(1, n, \min (n, 2-n), U) \\
& =\left\{\begin{array}{ll}
\epsilon_{0}(n) & \text { for } 0 \leqslant n \leqslant 1 \\
\epsilon_{0}(n-1)+U(n-1) & \text { for } 1 \leqslant n \leqslant 2
\end{array},\right.
\end{aligned}
$$

where $\epsilon_{0}\left(n_{\sigma}\right)=2 \int_{0}^{n_{\sigma} / 2} d k \epsilon_{k}$ is the kinetic energy of one spin species for the uncorrelated state. For the case of nearestneighbor hopping we have $\epsilon_{0}\left(n_{\sigma}\right)=-2 t \sin \left(\pi n_{\sigma}\right) / \pi, t>0$.
In Fig. 6 the exact ground-state energy, $E(n, m=0, U)$, obtained from the Bethe-ansatz solution, ${ }^{24}$ is compared to the Gutzwiller energy for zero and maximal polarization, at various densities. We also show the energy of the variational Hartree-Fock theory, $E_{\mathrm{HF}}(n, m=0, U)$; it is contained as a special case in the results for the GWF,

$E_{\mathrm{HF}}(n, m, U)=E_{\mathrm{G}}(1, n, m, U)$

$$
=\epsilon_{0}\left(\frac{n+m}{2}\right)+\epsilon_{0}\left(\frac{n-m}{2}\right)+\frac{U}{4}\left(n^{2}-m^{2}\right) .
$$

As expected, the g-optimized GWF significantly improves upon Hartree-Fock theory but overestimates $E$ at large $U .^{5}$ Since all spin configurations are degenerate for $U=\infty$, the exact ground-state energy coincides with $E_{\mathrm{FP}}$ in this case; therefore the Gutzwiller energy $E_{\mathrm{G}}^{\star}$ necessarily crosses the value $E_{\mathrm{FP}}$ at some finite value of the interaction $U_{c}$ (except for $n=1)$. The existence of a finite critical interaction $U_{c}$ above which the GWF predicts a ferromagnetic ground state is in contrast to the Lieb-Mattis theorem, ${ }^{25}$ which states that $m=0$ for the exact ground state (i.e., $U_{c}=\infty$ ). The reason for this overestimation of the instability of the paramagnetic state lies in the simple structure of the GWF, which controls only local correlations and cannot describe the special correlated behavior in $D=1$ microscopically.

The preceeding discussion only compared the variational energies for zero and full polarization. From our results for the ferromagnetic GWF we can also study the stability of partially polarized ferromagnetic states. We first consider Hartree-Fock theory. A simple calculation shows that it predicts a fully polarized ground state for $U \geqslant U_{c}^{\mathrm{HF}}(n)$, where

$$
U_{c}^{\mathrm{HF}}(n)=\left\{\begin{array}{ll}
16 \sin (\pi n / 2) & {[1-\cos (\pi n / 2)] /\left(\pi n^{2}\right)} \\
& \text { for } 0 \leqslant n \leqslant 1 \\
U_{c}^{\mathrm{HF}}(2-n) & \text { for } 1 \leqslant n \leqslant 2
\end{array} .\right.
$$

This critical interaction $U_{c}^{\mathrm{HF}}$ is smaller than that derived from the Stoner criterion, $\widetilde{U}_{c}^{\mathrm{HF}}(n)=1 / N\left(\epsilon_{n / 4}\right)=2 \pi \sin (\pi n / 2)$, where $N(\epsilon)$ is the density of states. Note that $E_{\mathrm{HF}}$ as a function of $m$ never develops a local minimum at $m \neq 0$. On the other hand, a maximum at finite $m$ occurs for $U>2[1$ $-\cos (\pi n)] / \min (n, 2-n)$, which leads to a global minimum at full polarization already for $U \geqslant U_{c}^{\mathrm{HF}}$. The Stoner criterion, which merely signals a negative curvature of $E_{\mathrm{HF}}$ at $m=0$ and does not take into account a finite magnetization, is thus irrelevant for the Hubbard chain with nearest-neighbor hopping.

For the Gutzwiller wave function we find that $E_{\mathrm{G}}^{\star}$ as a function of $m$ at fixed $U$ develops local extrema and global minima in a qualitatively similar fashion to $E_{\mathrm{HF}}$. As a consequence the GWF also describes a discontinuous transition from the paramagnetic state to a state with full polarization at $U=U_{c}(n)$. This critical interaction $U_{c}$ is shown in Fig. 7. Compared to Hartree-Fock theory we find agreement in the limit of small $n$. However, at intermediate densities the GWF predicts a significantly reduced ferromagnetic region. In particular for $n \rightarrow 1$ we have $U_{c} \rightarrow \infty$, as expected from the previous discussion [see also Fig. 6(a)]. Thus, in contrast to Hartree-Fock theory, the GWF does not exhibit a spurious ferromagnetic transition at half filling, since it is able to avoid double occupation not only through a ferromagnetic 


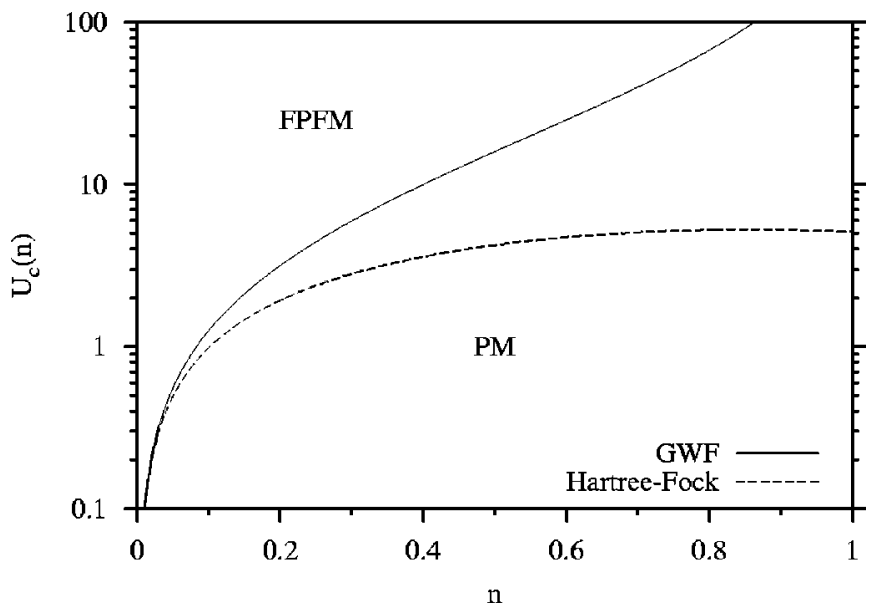

FIG. 7. Critical interaction $U_{c}(n)$ for the transition from a paramagnetic to a fully polarized ferromagnetic state for the Hubbard chain with nearest-neighbor hopping as obtained from the Gutzwiller wave function. The Hartree-Fock result, $U_{c}^{\mathrm{HF}}$ [Eq. (73)], is also shown; it has a shallow maximum at $n \approx 0.856$. Note that $U_{c}(n)=U_{c}(2-n)$.

polarization, but also by decreasing the variational parameter $g$. Away from half filling, however, the GWF predicts ferromagnetism for sufficiently large $U$, in contrast to the exact solution for the Hubbard chain.

\section{CONCLUSION}

In this paper we presented general diagrammatic relations for the expectation values of the $D$-dimensional Hubbard model in terms of the Gutzwiller wave function (GWF) at nonzero magnetization $m$. In $D=1$ explicit, approximationfree evaluations of the double occupation $d(g, n, m)$ and the momentum distribution $n_{k \sigma}(g, n, m)$ were made possible by exploiting (i) relations for the Feynman diagrams for $d$ and $n_{k \sigma}$, derived from canonical spin and particle-hole transformations, (ii) the polynomial form of the diagrams in powers of $k, n$, and $m$, and (iii) an analysis of the contributions of normal and umklapp processes. In this way the calculation of $d(g, n, m)$ and $n_{k \sigma}(g, n, m)$ was reduced to that for $m=0$. Furthermore, new closed expressions for the momentum distribution $n_{k \sigma}$ were derived, facilitating numerical evaluation.

The functions $d(g, n, m)$ and $n_{k \sigma}(g, n, m)$ in $D=1$ are qualitatively similar to those for $m=0$. The discontinuity $q_{\sigma}(g, n, m)$ of the momentum distribution at the Fermi energy was also calculated explicitly. It is always finite, except for the half filled band without double occupation $(g=0, n$ $=1, m=0$ ) in which case the electrons are trivially localized. In all other cases the GWF describes a ferromagnetic Fermi liquid.

Analysis of the Gutzwiller variational energy for the Hubbard chain with nearest-neighbor hopping shows that the GWF predicts a fully polarized ferromagnetic state at large enough $U$ and away from half filling, in contrast to the LiebMattis theorem. ${ }^{25}$ This exemplifies once more the peculiarities of the GWF which controls correlations between the electrons only globally through the local Hubbard interac- tion. While the GWF is an excellent wave function for the one-dimensional Heisenberg model (at least for $m=0),{ }^{6}$ since this only involves spin correlations between localized spins, it is not a very good wave function for the onedimensional Hubbard model at large $U$ and $n \neq 1$ since it does not describe density correlations well in this case. ${ }^{4}$ As a consequence the GWF cannot reproduce all characteristics of the one-dimensional system. This is also apparent from the finite discontinuity of the momentum distribution at the Fermi surface, which is, in fact, continuous for Luttinger liquids such as the one-dimensional Hubbard model. On the other hand the ferromagnetic GWF represents a trial state for partially polarized, itinerant electrons and may thus be regarded as an effective, nonperturbative description of a ferromagnetic Fermi liquid.

In view of the considerable technical complications involved in the present calculations it is not clear whether it will be possible to compute correlation functions with the GWF for $m \neq 0$. Since the calculation of the spin-spin correlation function ${ }^{6}$ for $m=0$ helped to gain considerable insight into the properties of Heisenberg-type models, ${ }^{7,8}$ a corresponding result for $m \neq 0$ would be helpful for a better understanding of one-dimensional Heisenberg models in a magnetic field.

\section{ACKNOWLEDGMENTS}

M.K. would like to acknowledge very helpful discussions with G.S. Uhrig in the early stages of this research. This work was supported in part by the Sonderforschungsbereich 484 of the Deutsche Forschungsgemeinschaft. Part of this work was carried out while M.K. was at Yale University, supported by DFG Grant KO 2056 and U.S. NSF Grant DMR 00-98226.

\section{APPENDIX A: CALCULATION OF POLYNOMIALS FOR THE NONMAGNETIC CASE}

In this appendix we describe the derivation of closed expressions for the polynomials $R_{p}(k)$ and $Q_{p}(k)$ that appear in Sec. III. By eliminating $Q_{p}(k)$ from MV's recursion formulas we obtain

$$
\begin{aligned}
p^{2} R_{p}(k)+p(p+1) R_{p+1}(k) & \\
= & k\left[(2 p-1) R_{p}^{\prime}(k)+2 p R_{p+1}^{\prime}(k)\right] \\
& -\left(k^{2}-\frac{1}{16}\right)\left[R_{p}^{\prime \prime}(k)+R_{p+1}^{\prime \prime}(k)\right], \quad p \geqslant 0 .
\end{aligned}
$$

Furthermore, the polynomials $Q_{p}(k)$ can be expressed in terms of $R_{p}(k)$ as

$$
\begin{aligned}
Q_{p+1}^{\prime}\left(k+\frac{1}{2}\right)= & -(2 p+1) R_{p}(k)-2(p+1) R_{p+1}(k) \\
& +\left(2 k-\frac{1}{2}\right)\left[R_{p}^{\prime}(k)+R_{p+1}^{\prime}(k)\right], \quad p \geqslant 0,
\end{aligned}
$$

together with $Q_{p}\left(\frac{3}{4}\right)=0$. We define $R_{0}(k)=1, Q_{0}(k)=0$.

A closed form for $R_{p}(k)$ is obtained as follows. Using Eqs. (56) and (57) we can reduce Eq. (A1) to 


$$
\begin{aligned}
& p(p+1) R_{p}(k)+2 k R_{p}^{\prime}(k)+\left(k^{2}-\frac{1}{16}\right) R_{p}^{\prime \prime}(k) \\
& \quad=p^{2} R_{p-1}(k), \quad p \geqslant 0 .
\end{aligned}
$$

This is essentially the differential equation of the Legendre polynomials except for the inhomogeneity on the right-hand side. From an expansion in Legendre polynomials $P_{n}(x)$ we thus obtain

$$
R_{p}(k)=\sum_{j=0}^{\lfloor p / 2\rfloor} \frac{(-1)^{p} p !^{2}(4 j+1)}{(p-2 j) !(p+2 j+1) !}\left(\begin{array}{c}
-\frac{1}{2} \\
j
\end{array}\right)^{2} P_{2 j}(4 k),
$$

which after some calculation yields Eq. (37). From $\mathcal{R}_{0}(x, z)$ we then obtain the explicit expressions in terms of a (terminating) hypergeometric function,

$$
\begin{aligned}
R_{p}(k) & =\sum_{j=0}^{\lfloor p / 2\rfloor} \frac{(-1)^{p}(2 p-2 j) !(2 j) !}{4^{j+p}(p-2 j) !(p-j) ! j !^{3}}\left(16 k^{2}-1\right)^{j} \\
& =\left(\begin{array}{c}
-\frac{1}{2} \\
p
\end{array}\right){ }_{3} F_{2}\left(-\frac{1}{2} p,-\frac{1}{2} p+\frac{1}{2}, \frac{1}{2} ; \frac{1}{2}-p, 1 ; 1-16 k^{2}\right) \\
& =\left(\begin{array}{c}
-\frac{1}{2} \\
p
\end{array}\right){ }_{3} F_{2}\left(-p,-p, \frac{1}{2} ; \frac{1}{2}-p, 1 ; \frac{4 k-1}{4 k+1}\right)\left(\frac{4 k+1}{2}\right)^{p} \\
& =\sum_{j=0}^{p}\left(\begin{array}{c}
p \\
j
\end{array}\right)\left(\begin{array}{c}
-\frac{1}{2} \\
j
\end{array}\right)\left(\begin{array}{c}
-\frac{1}{2} \\
p-j
\end{array}\right)\left(\frac{1-4 k}{2}\right)^{j}\left(\frac{1+4 k}{2}\right)^{p-j} .
\end{aligned}
$$

Furthermore, an integration by parts of Eq. (A2) leads to the expression for $\mathcal{Q}_{0}(x, z)$ in terms of $\mathcal{R}_{j}(x, z)$ shown in Eq. (38).

By using a hypergeometric identity to rewrite Eq. (37) as

$$
\begin{aligned}
\mathcal{R}_{0}(x, z)= & \frac{1}{\sqrt{1-z}}{ }_{2} F_{1}\left(\frac{1}{2}, \frac{1}{2} ; 1 ; \frac{\left(x^{2}-1\right) z^{2}}{4(1-z)}\right), \\
& \left|\frac{\left(1-x^{2}\right) z^{2}}{4(1-z)}\right|<1,
\end{aligned}
$$

integrating term wise with respect to $x$, and again using several hypergeometric identities we obtain the following explicit expression for $\mathcal{R}_{j}(x, z)$ :

$$
\mathcal{R}_{j}(x, z)=\frac{2^{2 j+1} j !}{(2 j) !} \sum_{p=0}^{\infty} \frac{(1-x)^{p+j} z^{2 p} \widetilde{R}_{p}^{(j)}(x)}{\left[(2-z)^{2}-(x z)^{2}\right]^{p+1 / 2}},
$$

where the $\widetilde{R}_{p}^{(j)}(x)$ are polynomials of degree $p$, (a)

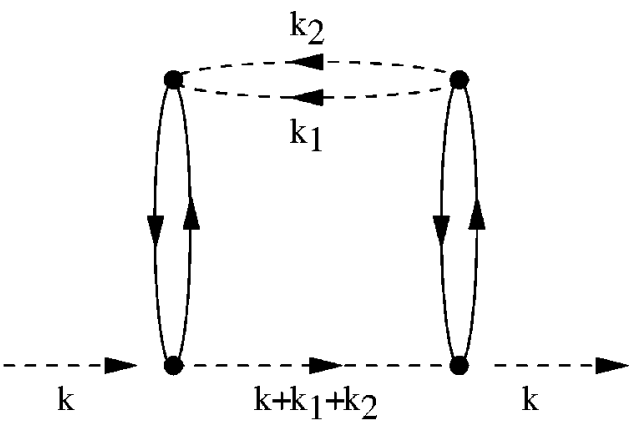

(b)

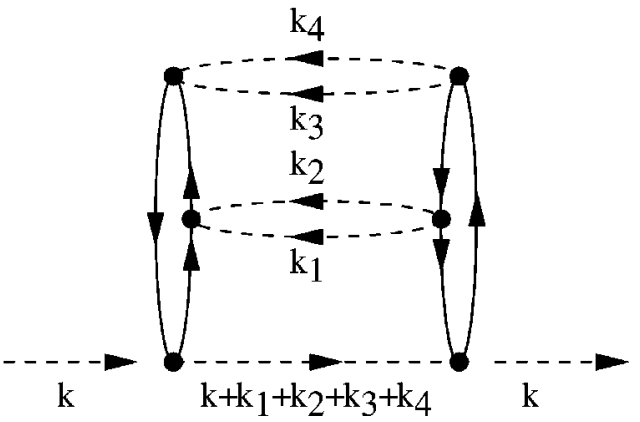

FIG. 8. A class of Feynman diagrams contributing to $h_{p \downarrow}^{>}(k, n, m)$. Solid (broken) lines represent majority (minority) spins and vanish unless they carry momentum in the interval $\left[-k_{\mathrm{F} \uparrow}, k_{\mathrm{F} \uparrow}\right]\left(\left[-k_{\mathrm{F} \downarrow}, k_{\mathrm{F} \downarrow}\right]\right)$; see MV for diagrammatic rules. For $k$ $>3 k_{\mathrm{F} \downarrow}$ the diagram in (a) vanishes since $k+k_{1}+k_{2} \in\left[-k_{\mathrm{F} \downarrow}, k_{\mathrm{F} \downarrow}\right]$ cannot be fulfilled. For $k>5 k_{\mathrm{F} \downarrow}$ the diagram in (b) vanishes for similar reasons, and so on for higher odd multiples of $k_{\mathrm{F} \downarrow}$.

$$
\begin{aligned}
\widetilde{R}_{p}^{(j)}(x)= & \left(\begin{array}{c}
-\frac{1}{2} \\
p
\end{array}\right)\left(\begin{array}{c}
-\frac{1}{2} \\
p+j
\end{array}\right) \\
& \times{ }_{3} F_{2}\left(-p, \frac{1}{2}, j ; 1+p+j, \frac{1}{2}+j ; \frac{1-x}{1+x}\right)(1+x)^{p} .
\end{aligned}
$$

The functions $\mathcal{R}_{j}(x, z)$ may be evaluated via the series (A10) for not too large values of $z$. Alternatively, the integration in Eq. (40) can be performed numerically.

Finally, we note a few special values. From MV's polynomial relations we obtain

$$
\begin{aligned}
\mathcal{Q}_{0}(x, z) & +\mathcal{Q}_{0}(-x, z) \\
& =1-(1-z) \mathcal{R}_{0}(x, z)+\frac{1}{2} \ln (1-z) .
\end{aligned}
$$

Together with Eqs. (37)-(39) we find in particular

$$
\mathcal{R}_{0}( \pm 1, z)=\frac{1}{\sqrt{1-z}}, \quad \mathcal{Q}_{0}(1, z)=0
$$

$$
\mathcal{Q}_{0}(-1, z)=1-\sqrt{1-z}+\frac{1}{2} \ln (1-z) .
$$


Furthermore, the sum rule for particles outside of the Fermi surface [Eq. (8)] implies

$$
\begin{aligned}
& \mathcal{R}_{1}(-1, z)=2 \mathcal{R}_{1}(0, z)=\frac{2}{z} \ln (1-z), \\
& \mathcal{Q}_{1}(-1, z)=-1+\frac{z-2}{2 z} \ln (1-z) .
\end{aligned}
$$

\section{APPENDIX B: POLYNOMIAL STRUCTURE \\ OF MINORITY SPIN DIAGRAMS OUTSIDE OF THE FERMI SURFACE}

In this appendix we derive the polynomial structure of the diagrams $h_{p \downarrow}^{>}$, for which many cases must be distinguished. As discussed at the beginning of Sec. III C, different polynomials occur in the contribution of normal processes depending on the relation of $k$ to $2 k_{\uparrow}-k_{\downarrow}$ and $2 k_{\uparrow}+k_{\downarrow}$. Furthermore, due to a certain class of diagrams, shown in Fig. 8, different polynomials for $h_{p \downarrow}^{>} / n^{p}$ may in principle occur also at $3 k_{\mathrm{F} \downarrow}, 5 k_{\mathrm{F} \downarrow}$, and all higher odd multiples of $k_{\mathrm{F} \downarrow}$; however, some simplification takes place, as we will show below.

Case $A: 0 \leqslant m \leqslant n / 3$. Here the momenta $2 k_{\uparrow}-k_{\downarrow}, 2 k_{\uparrow}$ $+k_{\downarrow}, 3 k_{\downarrow}$, etc., are ordered as follows:

$$
\frac{n+3 m}{4} \leqslant \frac{3(n-m)}{4}<\frac{3 n+m}{4} \leqslant \frac{5(n-m)}{4}
$$

so that the contribution of normal processes to $h_{p \downarrow}^{>}$, as it appears in Eq. (62), can be written in terms of three polynomials $A_{p}^{(i)}(k, m)$ of order $p$ :

$$
\begin{aligned}
& N_{p}\left(k, 0 \leqslant m \leqslant \frac{1}{3}\right) \\
& =\left\{\begin{array}{ll}
A_{p}^{(1)}(k, m) & \text { for } \frac{1-m}{4}<k \leqslant \frac{1+3 m}{4} \\
A_{p}^{(2)}(k, m) & \text { for } \frac{1+3 m}{4}<k \leqslant \frac{3(1-m)}{4} \\
A_{p}^{(3)}(k, m) & \text { for } \frac{3(1-m)}{4}<k \leqslant \frac{3+m}{4} \\
0 & \text { for } \frac{3+m}{4} \leqslant k
\end{array} .\right.
\end{aligned}
$$

If $k>1-(3 n+m) / 4$ umklapp processes contribute to $h_{p \downarrow}^{>}$ with momentum $1-k$, and several cases must be distinguished to determine the appropriate polynomials. We find for $n+m \leqslant 1$ and $m \geqslant 3 n-2$

$$
\frac{h_{p \downarrow}^{>}\left(k, n, 0 \leqslant m \leqslant \frac{n}{3}\right)}{n^{p}}= \begin{cases}A_{p}^{(1)}\left(\frac{k}{n}, \frac{m}{n}\right) & \text { for } \frac{n-m}{4}<k \leqslant \frac{n+3 m}{4} \\ A_{p}^{(2)}\left(\frac{k}{n}, \frac{m}{n}\right) & \text { for } \frac{n+3 m}{4}<k \leqslant \frac{3(n-m)}{4} \\ A_{p}^{(3)}\left(\frac{k}{n}, \frac{m}{n}\right) & \text { for } \frac{3(n-m)}{4}<k \leqslant \frac{1}{2}-\left|\frac{3 n+m}{4}-\frac{1}{2}\right|, \\ A_{p}^{(3)}\left(\frac{k}{n}, \frac{m}{n}\right)+A_{p}^{(3)}\left(\frac{1-k}{n}, \frac{m}{n}\right) & \text { for } 1-\frac{3 n+m}{4}<k \leqslant \frac{1}{2} \\ 0 & \text { for } \frac{3 n+m}{4}<k \leqslant \frac{1}{2}\end{cases}
$$

while for $n+m \leqslant 1$ and $m \leqslant 3 n-2$

$$
\frac{h_{p \downarrow}^{>}\left(k, n, 0 \leqslant m \leqslant \frac{n}{3}\right)}{n^{p}}= \begin{cases}A_{p}^{(1)}\left(\frac{k}{n}, \frac{m}{n}\right) & \text { for } \frac{n-m}{4}<k \leqslant \frac{n+3 m}{4} \\ A_{p}^{(2)}\left(\frac{k}{n}, \frac{m}{n}\right) & \text { for } \frac{n+3 m}{4}<k \leqslant 1-\frac{3 n+m}{4} \\ A_{p}^{(2)}\left(\frac{k}{n}, \frac{m}{n}\right)+A_{p}^{(3)}\left(\frac{1-k}{n}, \frac{m}{n}\right) & \text { for } 1-\frac{3 n+m}{4}<k \leqslant \frac{1}{2}-\left|\frac{3(n-m)}{4}-\frac{1}{2}\right| \\ A_{p}^{(2)}\left(\frac{k}{n}, \frac{m}{n}\right)+A_{p}^{(2)}\left(\frac{1-k}{n}, \frac{m}{n}\right) & \text { for } 1-\frac{3(n-m)}{4}<k \leqslant \frac{1}{2} \\ A_{p}^{(3)}\left(\frac{k}{n}, \frac{m}{n}\right)+A_{p}^{(3)}\left(\frac{1-k}{n}, \frac{m}{n}\right) & \text { for } \frac{3(n-m)}{4}<k \leqslant \frac{1}{2}\end{cases}
$$

whereas for $n+m \geqslant 1$ 


$$
\frac{h_{p \downarrow}^{>}\left(k, n, 0 \leqslant m \leqslant \frac{n}{3}\right)}{n^{p}}= \begin{cases}A_{p}^{(1)}\left(\frac{k}{n}, \frac{m}{n}\right) & \text { for } \frac{n-m}{4}<k \leqslant 1-\frac{3 n+m}{4} \\ A_{p}^{(1)}\left(\frac{k}{n}, \frac{m}{n}\right)+A_{p}^{(3)}\left(\frac{1-k}{n}, \frac{m}{n}\right) & \text { for } 1-\frac{3 n+m}{4}<k \leqslant \frac{n+3 m}{4} \\ A_{p}^{(2)}\left(\frac{k}{n}, \frac{m}{n}\right)+A_{p}^{(3)}\left(\frac{1-k}{n}, \frac{m}{n}\right) & \text { for } \frac{n+3 m}{4}<k \leqslant \frac{1}{2}-\left|\frac{3(n-m)}{4}-\frac{1}{2}\right| . \\ A_{p}^{(2)}\left(\frac{k}{n}, \frac{m}{n}\right)+A_{p}^{(2)}\left(\frac{1-k}{n}, \frac{m}{n}\right) & \text { for } 1-\frac{3(n-m)}{4}<k \leqslant \frac{1}{2} \\ A_{p}^{(3)}\left(\frac{k}{n}, \frac{m}{n}\right)+A_{p}^{(3)}\left(\frac{1-k}{n}, \frac{m}{n}\right) & \text { for } \frac{3(n-m)}{4}<k \leqslant \frac{1}{2}\end{cases}
$$

Case $B: n / 3 \leqslant m \leqslant n / 2$. Here on the other hand, we have the ordering

$$
\frac{3(n-m)}{4} \leqslant \frac{n+3 m}{4} \leqslant \frac{5(n-m)}{4} \leqslant \frac{3 n+m}{4} \leqslant \frac{7(n-m)}{4},
$$

and the contributions of normal processes to $h_{p \downarrow}^{>}$are now given by four polynomials $B_{p}^{(i)}(k, m)$ of order $p$ :

$$
N_{p}\left(k, \frac{1}{3} \leqslant m \leqslant \frac{1}{2}\right)=\left\{\begin{array}{ll}
B_{p}^{(1)}(k, m) & \text { for } \frac{1-m}{4} \leqslant k \leqslant \frac{1+3 m}{4} \\
B_{p}^{(2)}(k, m) & \text { for, } \frac{3(1-m)}{4} \leqslant k \leqslant \frac{1+3 m}{4} \\
B_{p}^{(3)}(k, m) & \text { for } \frac{1+3 m}{4} \leqslant k \leqslant \frac{5(1-m)}{4} . \\
B_{p}^{(4)}(k, m) & \text { for } \frac{5(1-m)}{4} \leqslant k \leqslant \frac{3+m}{4} \\
0 & \text { for } \frac{3+m}{4} \leqslant k
\end{array} .\right.
$$

Let us determine the umklapp process contributions for the region $1-n \leqslant m \leqslant n-1 / 2$,

$$
\frac{h_{p \downarrow}^{>}\left(k, n, \frac{n}{3} \leqslant m \leqslant \frac{n}{2}\right)}{n^{p}}=\left\{\begin{array}{ll}
B_{p}^{(1)}\left(\frac{k}{n}, \frac{m}{n}\right) & \text { for } \frac{n-m}{4}<k \leqslant 1-\frac{3 n+m}{4} \\
B_{p}^{(1)}\left(\frac{k}{n}, \frac{m}{n}\right)+B_{p}^{(4)}\left(\frac{1-k}{n}, \frac{m}{n}\right) & \text { for } 1-\frac{3 n+m}{4}<k \leqslant 1-\frac{5(n-m)}{4} \\
B_{p}^{(1)}\left(\frac{k}{n}, \frac{m}{n}\right)+B_{p}^{(3)}\left(\frac{1-k}{n}, \frac{m}{n}\right) & \text { for } 1-\frac{5(n-m)}{4}<k \leqslant \frac{3(n-m)}{4} \\
B_{p}^{(2)}\left(\frac{k}{n}, \frac{m}{n}\right)+B_{p}^{(3)}\left(\frac{1-k}{n}, \frac{m}{n}\right) & \text { for } \frac{3(n-m)}{4}<k \leqslant \frac{1}{2}-\left|\frac{n+3 m}{4}-\frac{1}{2}\right| \\
B_{p}^{(3)}\left(\frac{k}{n}, \frac{m}{n}\right)+B_{p}^{(3)}\left(\frac{1-k}{n}, \frac{m}{n}\right) & \text { for } \frac{n+3 m}{4}<k \leqslant \frac{1}{2} \\
B_{p}^{(2)}\left(\frac{k}{n}, \frac{m}{n}\right)+B_{p}^{(2)}\left(\frac{1-k}{n}, \frac{m}{n}\right) & \text { for } 1-\frac{n+3 m}{4}<k \leqslant \frac{1}{2}
\end{array} .\right.
$$

Now we connect the polynomials with one another via Eq. (61), which performs the transformation $n \rightarrow 1-m$ and $m$ $\rightarrow 1-n$. This provides a link between Eqs. (B4) and (B5), the right-hand sides of which are thus related, line by line, via Eq. (61). Similarly, Eqs. (B8) and (B3) are connected, which reveals that the distinction at momentum $1-5(n$ $-m) / 4$ in Eq. (B8) is in fact absent in Eq. (B3). Hence we find $B_{p}^{(3)}(k, m)=B_{p}^{(4)}(k, m)$, and also, by comparison with the first transformation, $B_{p}^{(1)}(k, m)=A_{p}^{(1)}(k, m), B_{p}^{(3)}(k, m)$ $=A_{p}^{(3)}(k, m)$. By inspecting the region $2 n-1 \leqslant m \leqslant 1-n$ of 
case $B$, we find a similar connection to larger $m$ (i.e., case C: $n / 2 \leqslant \mathrm{~m} \leqslant 3 n / 5$ ), and use of Eq. (61) shows that the new distinction at momentum $7(n-m) / 4$ disappears in a similar fashion, and that all its polynomials likewise reduce to the ones above. It is not difficult to see that this simplification repeats for larger magnetization, i.e., also for all $3 n / 5 \leqslant m \leqslant n$. We conclude that new polynomials at higher odd multiples of $(n-m) / 4$ are ruled out by the symmetry of the diagrams; only the distinction at momentum $3(n-m) / 4$ survives. Therefore the contribution (62) of normal processes to $h_{p \downarrow}^{>}$can finally be written as

$$
N_{p}(k, m)= \begin{cases}A_{p}^{(1)}(k, m) & \text { for } \frac{1-m}{4}<k \leqslant \min \left(\frac{1+3 m}{4}, \frac{3(1-m)}{4}\right) \\ A_{p}^{(2)}(k, m) & \text { for } m \leqslant \frac{1}{3} \text { and } \frac{1+3 m}{4} \leqslant k \leqslant \frac{3(1-m)}{4} \\ B_{p}^{(2)}(k, m) & \text { for } m \geqslant \frac{1}{3} \text { and } \frac{3(1-m)}{4} \leqslant k \leqslant \frac{1+3 m}{4}, \\ A_{p}^{(3)}(k, m) & \text { for } \max \left(\frac{1+3 m}{4}, \frac{3(1-m)}{4}\right) \leqslant k \leqslant \frac{3+m}{4} \\ 0 & \text { for } \frac{3+m}{4} \leqslant k\end{cases}
$$

i.e., a total of four polynomials are needed to describe $n_{k \downarrow}^{>}$; they are determined in Appendix C.

\section{APPENDIX C: \\ CALCULATION OF POLYNOMIALS FOR MINORITY SPINS OUTSIDE OF THE FERMI SURFACE}

In this appendix we determine the polynomials that appear on the right-hand side of Eq. (B9). First let us examine $A_{p}^{(2)}(k, m)$. For $m=0$ we immediately obtain $A_{p}^{(2)}(k, 0)$ $=Q_{p}(k)$ by comparison with Eq. (31). Furthermore, we can derive its behavior for small $m$ from the equation

$$
h_{p \downarrow}^{>}(k, n, m)=h_{p \uparrow}^{>}(k, n,-m),
$$

which is a simple consequence of Eq. (10b). Similar to MV it can be shown that $h_{p \uparrow}(k, n, m)$, when regarded as a function of $n$, has two continuous derivatives at $n=1$ (for all $k$ $\left.\neq k_{\mathrm{F} \uparrow}\right)$. Then Eq. (49) implies that $h_{p \uparrow}(k, n, m)$ has the same property as a function of $m$ at $m=0$. Hence the expression (50) may be used on the right-hand side of Eq. (C1) for small positive $m$, up to an error of $O\left(\mathrm{~m}^{3}\right)$. Inserting the appropriate polynomials for momenta in the interval $(n+3 m) / 4<k$ $<\min [3(n-m) / 4,1-(3 n+m) / 4]$ we obtain

$$
\begin{aligned}
A_{p+2}^{(2)}(k, m)= & \sum_{r=0}^{p}\left(\begin{array}{c}
p+1 \\
r+1
\end{array}\right)(1+m)^{r+2} m^{p-r} \\
& \times Q_{r+2}\left(\frac{k+m / 2}{1+m}\right)+O\left(m^{3}\right),
\end{aligned}
$$

which holds for all $k$ and small $m$.

The following definitions will help keep the notation compact. Let $P$ be any of $A^{(1)}, A^{(2)}, B^{(2)}, A^{(3)}$. In addition to the polynomials $P_{p}(x, y)$, we define $\widetilde{P}_{p}(x, y)=P_{p}(x, y)$ $+c_{p-1}(1, y)$, and introduce their generating functions

$$
\begin{gathered}
P(x, y, z)=\sum_{p=2}^{\infty} P_{p}(x, y)(-z)^{p}, \\
\widetilde{P}^{(\alpha)}(x, y, z)=P(x, y, z)-\frac{z(1-y)}{2}+\frac{1}{2} \ln \frac{1-y z}{1-z} .
\end{gathered}
$$

Now we are ready to collect the relations between Eqs. (B4) and (B5) and between Eqs. (B8) and (B3) that Eq. (61) provides (see Appendix B). Setting $x=k / n, y=m / n$, and $s$ $=1 / n$ in these relations, we obtain (for arbitrary $s$ )

$$
\begin{aligned}
& \widetilde{A}_{p+2}^{(1)}(x, y)=\sum_{r=0}^{p}\left(\begin{array}{l}
p \\
r
\end{array}\right)(s-y)^{r+2}(-s)^{p-r} \widetilde{A}_{r+2}^{(1)}\left(\frac{x}{s-y}, \frac{s-1}{s-y}\right), \\
& =\sum_{r=0}^{p}\left(\begin{array}{l}
p \\
r
\end{array}\right)(s-y)^{r+2}(-s)^{p-r} \widetilde{A}_{r+2}^{(2)}\left(\frac{x}{s-y}, \frac{s-1}{s-y}\right),
\end{aligned}
$$

$$
\begin{aligned}
& \widetilde{B}_{p+2}^{(2)}(x, y)+A_{p+2}^{(3)}(s-x, y) \\
& \quad=\sum_{r=0}^{p}\left(\begin{array}{l}
p \\
r
\end{array}\right)(s-y)^{r+2}(-s)^{p-r} \widetilde{A}_{r+2}^{(3)}\left(\frac{x}{s-y}, \frac{s-1}{s-y}\right),
\end{aligned}
$$

$\widetilde{B}_{p+2}^{(2)}(x, y)+B_{p+2}^{(2)}(s-x, y)=-c_{p+1}(1, y)-s c_{p}(1, y)$, 
and other relations that are in fact implied by these. By repeated combination of Eqs. (C5) we find the important equality

$$
A_{p}^{(1)}(x, y)+A_{p}^{(3)}(x, y)=A_{p}^{(2)}(x, y)+B_{p}^{(2)}(x, y) .
$$

Furthermore, by setting $s=2 x$ in Eq. (C5c) we immediately obtain the explicit expression

$$
B_{p+2}^{(2)}(x, y)=-c_{p+1}(1, y)-x c_{p}(1, y) .
$$

In terms of generating functions our results so far can be expressed as

$$
\begin{gathered}
A^{(2)}(x, y, z)=\mathcal{Q}_{0}\left(\frac{4 x-2}{1+y}, \frac{(1+y) z}{1+y z}\right)+O\left(y^{3}\right), \\
\widetilde{A}^{(1)}(x, y, z)=(1-s z) \widetilde{A}^{(1)}\left(\frac{x}{s-y}, \frac{s-1}{s-y}, \frac{(s-y) z}{s z-1}\right),
\end{gathered}
$$

$\widetilde{A}^{(1)}(x, y, z)+\widetilde{A}^{(3)}(s-x, y, z)$

$$
=(1-s z) \widetilde{A}^{(2)}\left(\frac{x}{s-y}, \frac{s-1}{s-y}, \frac{(s-y) z}{s z-1}\right),
$$

$$
\begin{aligned}
\widetilde{B}^{(2)}(x, y, z) & =(1-s z) \widetilde{B}^{(2)}\left(\frac{x}{s-y}, \frac{s-1}{s-y}, \frac{(s-y) z}{s z-1}\right) \\
& =\frac{x z}{2} \ln \frac{1-y z}{1-z},
\end{aligned}
$$

$\widetilde{A}^{(3)}(x, y, z)=\widetilde{B}^{(2)}(x, y, z)$

$$
+(1-s z) \tilde{A}^{(3)}\left(\frac{s-x}{s-y}, \frac{s-1}{s-y}, \frac{(s-y) z}{s z-1}\right) .
$$

We proceed to determine $A^{(3)}$. First we obtain $A^{(3)}(x, 0, z)$ from Eq. (C10) at $y=0$ by inserting the expansion $(\mathrm{C} 8)$ and differentiating with respect to $s$; we then set $s=1$, so that the error term vanishes. This yields an expression for $d A^{(3)}(x, 0, z) / d x$, which we integrate with respect to $x$, using $A^{(3)}(3 / 4,0, z)=0$. This result for $A^{(3)}(x, 0, z)$ is used in Eq. (C12) with $s=1$, which yields $A^{(3)}(x, y, z)$ for arbitrary $y$, $A^{(3)}(x, y, z)$

$$
\begin{aligned}
= & \frac{(1-y z)(4 x-1-3 y)}{2(1-y)} \mathcal{Q}_{0}\left(\frac{4 x-2-2 y}{1-y}, \frac{(1-y) z}{1-y z}\right) \\
& -\frac{(1-3 y) z+2}{4} \mathcal{Q}_{1}\left(\frac{4 x-2-2 y}{1-y}, \frac{(1-y) z}{1-y z}\right) \\
& -\frac{(1-y)(1-z) z}{2(1-y z)} \dot{\mathcal{Q}}_{1}\left(\frac{4 x-2-2 y}{1-y}, \frac{(1-y) z}{1-y z}\right) .
\end{aligned}
$$

The dot indicates derivative with respect to second argument. Next we derive $A^{(1)}$. First we obtain $A^{(1)}(x, 0, z)$ from Eq. (C10) at $s=1$ and $y=0$, using Eq. (C8) at $y=0$. This result for $A^{(1)}(x, 0, z)$ is used in Eq. (C9) with $s=1$, which thus yields $A^{(1)}(x, y, z)$ for arbitrary $y$,

$$
\begin{aligned}
A^{(1)}(x, y, z) & \\
= & \frac{(1-y z)(3-3 y-4 x)}{2(1-y)} \mathcal{Q}_{0}\left(\frac{4 x-2+2 y}{1-y}, \frac{(1-y) z}{1-y z}\right) \\
& +\frac{(1-3 y) z+2}{4} \mathcal{Q}_{1}\left(\frac{4 x-2+2 y}{1-y}, \frac{(1-y) z}{1-y z}\right) \\
& +\frac{(1-y)(1-z) z}{2(1-y z)} \dot{\mathcal{Q}}_{1}\left(\frac{4 x-2+2 y}{1-y}, \frac{(1-y) z}{1-y z}\right) \\
& +B^{(2)}(x, y, z) .
\end{aligned}
$$

Finally, $A^{(2)}(x, y, z)$ is obtained from Eq. (C6). These results can be rearranged into Eqs. (63)-(65).
*Email address: Marcus.Kollar@itp.uni-frankfurt.de; present address: Institut für Theoretische Physik, Johann-Wolfgang-GoetheUniversität Frankfurt, Robert-Mayer-Straße 8-10, D-60054 Frankfurt am Main, Germany.

${ }^{\dagger}$ Email address: Dieter.Vollhardt@physik.uni-augsburg.de

${ }^{1}$ J. Hubbard, Proc. R. Soc. London, Ser. A 276, 238 (1963).

${ }^{2}$ J. Kanamori, Prog. Theor. Phys. 30, 275 (1963).

${ }^{3}$ M. C. Gutzwiller, Phys. Rev. Lett. 10, 159 (1963); Phys. Rev. 134, A923 (1964); 137, A1726 (1965).

${ }^{4}$ D. Vollhardt, P. G. J. van Dongen, F. Gebhard, and W. Metzner, Mod. Phys. Lett. B 4, 499 (1990).

${ }^{5}$ W. Metzner and D. Vollhardt, Phys. Rev. Lett. 59, 121 (1987); Phys. Rev. B 37, 7382 (1988); 39, 12339 (1989).

${ }^{6}$ F. Gebhard and D. Vollhardt, Phys. Rev. Lett. 59, 1472 (1987); Phys. Rev. B 38, 6911 (1988).

${ }^{7}$ F. D. M. Haldane, Phys. Rev. Lett. 60, 635 (1988).

${ }^{8}$ B. S. Shastry, Phys. Rev. Lett. 60, 639 (1988).

${ }^{9}$ H. Yokoyama and H. Shiba, J. Phys. Soc. Jpn. 56, 1490 (1987)

${ }^{10}$ H. Yokoyama and S. Tokizaki, Physica B 230, 418 (1997).

${ }^{11}$ Z. Gulácsi and M. Gulácsi, Phys. Rev. B 44, 1475 (1991).

${ }^{12}$ Z. Gulácsi, M. Gulácsi, and B. Jankó, Phys. Rev. B 47, 4168
(1993).

${ }^{13}$ W. Metzner and D. Vollhardt, Phys. Rev. Lett. 62, 324 (1989).

${ }^{14}$ F. Gebhard, Phys. Rev. B 41, 9452 (1990).

${ }^{15}$ J. Bünemann, W. Weber, and F. Gebhard, Phys. Rev. B 57, 6896 (1998).

${ }^{16}$ W. Weber, J. Bünemann, and F. Gebhard, in Band-Ferromagnetism: Ground-State and Finite-Temperature Phenomena, edited by K. Baberschke, M. Donath, and W. Nolting, Lecture Notes in Physics, Vol. 580 (Springer, Heidelberg, 2001), p. 9.

${ }^{17}$ W. F. Brinkman and T. M. Rice, Phys. Rev. B 2, 4302 (1970).

${ }^{18}$ M. Kollar and D. Vollhardt, Phys. Rev. B 63, 045107 (2001).

${ }^{19}$ D. Vollhardt, N. Blümer, K. Held, M. Kollar, J. Schlipf, M. Ulmke, and J. Wahle, Adv. Solid State Phys. 38, 383 (1999).

${ }^{20}$ Note that for nonisotropic Fermi surfaces, in particular in dimension $D>1, q_{\sigma}$ may depend not only on the magnitude but also on the direction of $\boldsymbol{k}_{F \sigma}$.

${ }^{21}$ K. Hashimoto, Phys. Rev. B 31, 7368 (1985).

${ }^{22}$ H. S. Wilf, generatingfunctionology (Academic, San Diego, 1994).

${ }^{23}$ M. Kollar, cond-mat/0105068 (unpublished).

${ }^{24}$ E. H. Lieb and F. Y. Wu, Phys. Rev. Lett. 20, 1445 (1968).

${ }^{25}$ E. H. Lieb and D. Mattis, Phys. Rev. 125, 164 (1962). 